\title{
Nonlinear Screening and Effective Electrostatic Interactions in Charge-Stabilized Colloidal Suspensions
}

\author{
A. R. Dentor * \\ Department of Physics, North Dakota State University, Fargo, ND, 58105-5566
}

(Dated: November 5, 2018)

\begin{abstract}
A nonlinear response theory is developed and applied to electrostatic interactions between spherical macroions, screened by surrounding microions, in charge-stabilized colloidal suspensions. The theory describes leading-order nonlinear response of the microions (counterions, salt ions) to the electrostatic potential of the macroions and predicts microion-induced effective many-body interactions between macroions. A linear response approximation [Phys. Rev. E 62, 3855 (2000)] yields an effective pair potential of screened-Coulomb (Yukawa) form, as well as a one-body volume energy, which contributes to the free energy. Nonlinear response generates effective many-body interactions and essential corrections to both the effective pair potential and the volume energy. By adopting a random-phase approximation (RPA) for the response functions, and thus neglecting microion correlations, practical expressions are derived for the effective pair and triplet potentials and for the volume energy. Nonlinear screening is found to weaken repulsive pair interactions, induce attractive triplet interactions, and modify the volume energy. Numerical results for monovalent microions are in good agreement with available $a b$ initio simulation data and demonstrate that nonlinear effects grow with increasing macroion charge and concentration and with decreasing salt concentration. In the dilute limit of zero macroion concentration, leading-order nonlinear corrections vanish. Finally, it is shown that nonlinear response theory, when combined with the RPA, is formally equivalent to the mean-field Poisson-Boltzmann theory and that the linear response approximation corresponds, within integral-equation theory, to a linearized hypernetted-chain closure.
\end{abstract}

\section{INTRODUCTION}

Electrostatic interactions between charged macromolecules dispersed in an electrolyte solvent have attracted sustained cross-disciplinary interest because of their fundamental role in governing the physical properties of colloidal suspensions [1, 2, 3], polyelectrolyte solutions [4, 5], and many biological systems. Colloids (nm- $\mu \mathrm{m}$-sized particles) and polyelectrolytes (charged polymers) can acquire charge in solution through dissociation of counterions. Familiar examples of charged colloids are latex or silica microspheres, clay platelets, and ionic micelles suspended in water. Common polyelectrolytes are polyacrylic acid, found in gels and rheology modifiers, and biopolymers (e.g., DNA, proteins, starches) in aqueous solution. In all of these systems, bare Coulomb interactions between charged macromolecules (macroions) are screened by counterions and salt ions (microions). This paper formulates a general response theory of microion screening and applies the theory to microion-induced effective pair and many-body interactions between colloidal macroions in suspension.

In recent years, experimental reports of apparent attractions between like-charged macroions have focused attention on electrostatic interactions in strongly charged, deionized suspensions. Observations of anomalous thermodynamic behavior, such as bulk phase separation [ 6, 7, [8, 9] and metastable crystallites [10], and direct measurements of attractive interactions between confined macroions [11, 12] have motivated a variety of theories and computer simulation methods, which have been recently reviewed [13, 14, 15, 16].

A variety of simulation methods have been applied to explore effective interparticle interactions and phase behavior in charged colloids. Standard molecular dynamics 17] and Monte Carlo [1, 19, 20] algorithms have been used to investigate crystallization in effective one-component pairwise-interacting systems, while powerful ab initio (classical Car-Parrinello) 21, 22] and multi-component Monte Carlo [23, 24, 25, 26] techniques have modeled effective interactions and, to a lesser degree, phase behavior.

*Electronic address: alan.denton@ndsu.nodak.edu 
Theoretical approaches can be broadly distinguished by the extent to which they include correlations between microions. Many approaches are founded on the Poisson-Boltzmann (PB) equation for the electrostatic potential, which is derived from mean-field approximations that neglect microion correlations. The classic theory of Derjaguin and Landau [27] and Verwey and Overbeek [28] (DLVO), based on a linearization of the PB equation, predicts that widely separated macroions interact via a purely repulsive effective electrostatic pair potential of screened-Coulomb (Yukawa) form. Similar effective interactions have been derived within the frameworks of density-functional (DF) theory [22, 29, 30, 31], response theory [32, 33, 34], and extended Debye-Hückel (DH) theories 35, 36 . These more recent approaches also clarify the importance of a one-body volume energy [29, 30, 31, 32, 33, 34, 35, 36], which contributes a state-dependent term to the free energy and thus can influence thermodynamic behavior.

Microion correlations, while often weak for monovalent microions, generally cannot be ignored in the case of multivalent microions, as emphasized in several recent studies of charged colloids and polyelectrolytes 25, 37. 38, 39, 40, 41]. Microion correlations can induce short-range attractions, which have been linked to condensation of DNA and other polyelectrolytes [37, 38, 39, 42]. Another wide class of theories that include some microion correlations is the class of integral-equation theories [43, 44, 45, 46, 47, 48, 49], which predict multi-component correlation functions from the Ornstein-Zernike relation combined with various closures.

Many theoretical approaches rely, in practice, on some manner of linear approximation. DLVO theory and linearized PB cell models [50, 51, 52] are based on the linearized PB equation. The DF [29, 30, 31] and response theory [32, 33, 34] approaches involve truncating expansions of free energy functionals or of microion density profiles. While linear approximations can be justified under a wide range of conditions, their validity may be questioned for concentrated suspensions of highly charged macroions at low salt concentrations (ionic strengths) - precisely those conditions under which anomalous phase behavior has been reported. On the other hand, many nonlinear theories, such as the full PB theory [53, 54, 55] and integral-equation theories, present severe computational challenges. In fact, the nonlinear PB equation usually yields to numerical solution only within cell models with simplified boundary conditions.

The main purpose of the present paper is to extend response theory to include leading-order nonlinear microion screening and to apply the extended theory to systematically test the linear-screening approximation. This extension necessarily entails three-body effective interactions between macroions and corrections at the pair and one-body levels, for which computationally practical expressions are derived. The predicted effective interactions could, in future studies, be input directly into statistical mechanical theories or simulations to study influences of nonlinear screening on phase equilibria and other phenomena.

The key qualitative conclusion of the paper is that nonlinear effects can significantly modify effective interactions, becoming increasingly important with increasing macroion charge and concentration and with decreasing salt concentration. Numerical calculations for bulk suspensions are performed to quantify parameter ranges wherein linearization is justified. Comparison is made with a similar extension of the DF approach, recently applied to wall-induced effective pair interactions [56, 57] and to effective triplet interactions [58].

Outlining the remainder of the paper, Sec. III defines the model colloidal suspension; Sec. III develops a general response theory for the system; Sec. IV presents analytical results for leading-order nonlinear corrections to the effective microion-induced interactions; Sec. V presents numerical results, for selected parameters, and comparisons with predictions of linear response theory; Sec. VI summarizes the paper; and finally the Appendix compares response theory with two related approaches, namely PB theory and integral-equation theory.

\section{MODEL}

The system of interest comprises colloidal macroions, counterions, and salt ions dispersed in a solvent (Fig. 1). This multi-component mixture is modeled here as a collection of $N_{m}$ charged hard-sphere macroions, of valence $-Z$ (surface charge $-Z e$ ) and radius $a$ (diameter $\sigma=2 a$ ), and $N_{c}$ point counterions of valence $z$ in an electrolyte solvent of volume $V$ at temperature $T$. Global charge neutrality constrains macroion and counterion numbers via the relation $Z N_{m}=z N_{c}$. For simplicity, we assume a symmetric electrolyte consisting of $N_{s}$ point salt ions of valence $z$ and $N_{s}$ of valence $-z$ (same valence as counterions) in a uniform solvent. The microions thus number $N_{+}=N_{c}+N_{s}$ positive and $N_{-}=N_{s}$ negative, for a total of $N_{\mu}=N_{c}+2 N_{s}$. The solvent is approximated, within the primitive model, as a dielectric continuum, characterized entirely by a dielectric constant $\epsilon$. 
The macroion charge, which may be physically interpreted as an effective (renormalized) charge, is assumed to be fixed and distributed smoothly over the particle surface. Charge discreteness can be reasonably neglected if the distance between neighboring macroion surfaces much exceeds the typical distance between charge groups on a macroion surface, roughly $\sigma / \sqrt{Z}$. The assumption of point microions limits the model to systems with large size asymmetries. Furthermore, we neglect polarization effects, e.g., charge-induced dipole interactions [59, 60, 61], which are shorter-ranged than charge-charge interactions, and which vanish if solvent and macroions have the same dielectric constant (i.e., are index matched).

\section{THEORY}

\section{A. Reduction to One Component}

The response theory of effective interactions is fundamentally based on a reduction of the multi-component mixture to an equivalent one-component system by integrating out the degrees of freedom of the microions 62. In this reduction, the macroions are regarded as applying an "external" potential that perturbs the (otherwise uniform) microion distribution. For a sufficiently weak perturbation (dilute or weakly charged macroions), the microions respond linearly. The linear response approximation has been discussed in refs. 32, 33, 34]. Upon increasing the macroion charge or concentration, however, nonlinear microion response becomes increasingly important. This motivates the current extension of response theory from linear to nonlinear response.

To simplify the derivation, we first consider salt-free suspensions and introduce salt ions only at the end. The model system is then described by a Hamiltonian $H$ that decomposes naturally into three terms:

$$
H=H_{m}(\{\mathbf{R}\})+H_{c}(\{\mathbf{r}\})+H_{m c}(\{\mathbf{R}\},\{\mathbf{r}\}),
$$

where $\{\mathbf{R}\}$ and $\{\mathbf{r}\}$ denote the coordinates of macroions and microions, respectively. The first term on the right side of Eq. (10) is the bare macroion Hamiltonian, given by

$$
H_{m}=H_{\mathrm{HS}}(\{\mathbf{R}\})+\frac{1}{2} \sum_{i \neq j=1}^{N_{m}} v_{m m}\left(\left|\mathbf{R}_{i}-\mathbf{R}_{j}\right|\right),
$$

where $H_{\mathrm{HS}}$ is the Hamiltonian for neutral hard spheres (the macroion hard cores) and $v_{m m}(r)=Z^{2} e^{2} / \epsilon r$, $r>\sigma$, is the bare Coulomb pair interaction between macroions. In the primitive model, the solvent acts only to reduce the strength of Coulomb interactions by a factor $1 / \epsilon$. The second term of the Hamiltonian,

$$
H_{c}=K_{c}+\frac{1}{2} \sum_{i \neq j=1}^{N_{c}} v_{c c}\left(\left|\mathbf{r}_{i}-\mathbf{r}_{j}\right|\right)
$$

describes the counterions alone, having kinetic energy $K_{c}$ and interacting via a Coulomb pair potential $v_{c c}(r)=z^{2} e^{2} / \epsilon r$. The third term is the macroion-counterion interaction energy:

$$
H_{m c}=\sum_{i=1}^{N_{m}} \sum_{j=1}^{N_{c}} v_{m c}\left(\left|\mathbf{R}_{i}-\mathbf{r}_{j}\right|\right)
$$

where $v_{m c}(r)$ is the macroion-counterion electrostatic pair interaction: $v_{m c}(r)=Z z e^{2} / \epsilon r, r>a$. For impenetrable hard-core macroions, the form of $v_{m c}(r)$ inside the core is arbitrary and can be specified so as to minimize counterion penetration inside the cores (see Sec. IVA). For later reference, we note that the macroion and counterion Hamiltonians [Eqs. (2) and (3)] may be expressed in terms of Fourier components using the identity

$$
\sum_{i \neq j=1}^{N} v\left(\left|\mathbf{r}_{i}-\mathbf{r}_{j}\right|\right)=\frac{1}{V} \sum_{\mathbf{k}} \hat{v}(k)[\hat{\rho}(\mathbf{k}) \hat{\rho}(-\mathbf{k})-N]
$$


where $\hat{v}(k)$ is the Fourier transform of a pair potential $v(r), \hat{\rho}(\mathbf{k})$ is the Fourier transform of the appropriate (macroion or counterion) number density operator $\rho(\mathbf{r})=\sum_{i=1}^{N} \delta\left(\mathbf{r}-\mathbf{r}_{i}\right)$, and the Fourier transform convention is

$$
\begin{aligned}
& \hat{\rho}(\mathbf{k})=\int \mathrm{d} \mathbf{r} \rho(\mathbf{r}) e^{-i \mathbf{k} \cdot \mathbf{r}} \\
& \rho(\mathbf{r})=\frac{1}{V} \sum_{\mathbf{k}} \hat{\rho}(\mathbf{k}) e^{i \mathbf{k} \cdot \mathbf{r}} .
\end{aligned}
$$

The inverse transform is expressed as a summation, rather than an integral, in anticipation that charge neutrality will necessitate singling out the $k=0$ component for special treatment.

At constant temperature and volume, the thermodynamic behavior of the system is governed by the canonical partition function,

$$
\mathcal{Z}=\left\langle\langle\exp (-\beta H)\rangle_{c}\right\rangle_{m}
$$

where $\beta=1 / k_{B} T$ and \langle\rangle$_{c}$ and \langle\rangle$_{m}$ denote classical traces over counterion and macroion coordinates, respectively. The two-component mixture of macroions and counterions can be formally mapped onto an equivalent one-component system of "pseudo-macroions" by performing a restricted trace over counterion coordinates, keeping the macroions fixed. Thus, without approximation,

$$
\mathcal{Z}=\left\langle\exp \left(-\beta H_{\mathrm{eff}}\right)\right\rangle_{m}
$$

where $H_{\text {eff }}=H_{m}+F_{c}$ is the effective Hamiltonian of the equivalent one-component system and

$$
F_{c}=-k_{B} T \ln \left\langle\exp \left[-\beta\left(H_{c}+H_{m c}\right)\right]\right\rangle_{c}
$$

can be interpreted as the free energy of a nonuniform gas of counterions in the presence of the fixed macroions. To simplify notation, we henceforth omit the subscript $c$ from the trace over counterion coordinates: \langle\rangle$_{c} \equiv$ \langle\rangle .

\section{B. Response Theory}

Although the one-component mapping is exact, the challenge now shifts to approximating the counterion free energy $F_{c}$. Progress can be made by regarding the macroions as an "external" potential for the counterions and invoking perturbation theory 32,63 :

$$
F_{c}=F_{0}+\int_{0}^{1} \mathrm{~d} \lambda\left\langle H_{m c}\right\rangle_{\lambda},
$$

where $F_{0}=-k_{B} T \ln \left\langle\exp \left(-\beta H_{c}\right)\right\rangle$ is the reference free energy of the counterions in the presence of neutral (hard-core) macroions (the counterions then being unperturbed, except for exclusion from the macroion cores), \langle\rangle$_{\lambda}$ denotes a trace over coordinates of the counterions in the presence of macroions charged to a fraction $\lambda$ of their full charge, and the $\lambda$-integral adiabatically charges the macroions from neutral to fully charged. Although each term on the right side of Eq. (11) is infinite, the infinities cancel to yield a finite counterion free energy. When the macroions are uncharged, the surrounding "sea" of counterions has uniform density, neglecting any confinement-induced structure, which is reasonable for typical counterion concentrations in colloidal suspensions (see below). As the macroion charge is "turned on," the counterions respond, redistributing themselves to form a double layer (surface charge plus neighboring counterions) surrounding each macroion.

In practice, it proves convenient to convert $F_{0}$ to the free energy of a classical one-component plasma (OCP) by adding and subtracting the energy of a uniform compensating negative background. The background energy can be expressed as $E_{b}=-\frac{1}{2} N_{c} n_{c} \hat{v}_{c c}(0)$, where $n_{c}$ is the average density of counterions in the volume unoccupied from the macroion cores. Note that the infinite background energy formally cancels the infinities 
on the right side of Eq. (11). Because the counterions are excluded (with the background) from the hard macroion cores, the OCP has average density $n_{c}=N_{c} / V^{\prime}$, where $\eta=\frac{\pi}{6}\left(N_{m} / V\right) \sigma^{3}$ is the macroion volume fraction and $V^{\prime}=V(1-\eta)$ is the free volume. Thus,

$$
F_{c}=F_{\mathrm{OCP}}+\int_{0}^{1} \mathrm{~d} \lambda\left\langle H_{m c}\right\rangle_{\lambda}-E_{b}
$$

where $F_{\mathrm{OCP}}=F_{0}+E_{b}$ is the free energy of the OCP in the presence of neutral, but volume-excluding, hard spheres - what might be loosely called a "Swiss cheese" OCP.

In terms of Fourier components, the macroion-counterion interaction can be expressed as

$$
\left\langle H_{m c}\right\rangle_{\lambda}=\frac{1}{V^{\prime}} \sum_{\mathbf{k}} \hat{v}_{m c}(k) \hat{\rho}_{m}(\mathbf{k})\left\langle\hat{\rho}_{c}(-\mathbf{k})\right\rangle_{\lambda} .
$$

Evidently, $\left\langle H_{m c}\right\rangle_{\lambda}$ depends through $\left\langle\hat{\rho}_{c}(\mathbf{k})\right\rangle_{\lambda}$ upon the response of the counterions to the macroion charge density. Note, however, that there is no response for $k=0$, since $\hat{\rho}_{c}(0)=\int \mathrm{d} \mathbf{r} \rho_{c}(\mathbf{r})=N_{c}$, which is fixed by charge neutrality for a given macroion concentration. Taking this $k \rightarrow 0$ limit into account, and subtracting the background energy, Eq. (13) becomes

$$
\left\langle H_{m c}\right\rangle_{\lambda}-E_{b}=\frac{1}{V^{\prime}} \sum_{\mathbf{k} \neq \mathbf{0}} \hat{v}_{m c}(k) \hat{\rho}_{m}(\mathbf{k})\left\langle\hat{\rho}_{c}(-\mathbf{k})\right\rangle_{\lambda}+n_{c} \lim _{k \rightarrow 0}\left[N_{m} \hat{v}_{m c}(k)+\frac{N_{c}}{2} \hat{v}_{c c}(k)\right] .
$$

To proceed further, we apply a perturbative approximation for the macroion-induced counterion density, adapting a standard approach from the theory of metals [63, 64, 65, 66]. Defining the macroion external potential $\phi_{\text {ext }}(\mathbf{r})$ by

$$
z e \phi_{\mathrm{ext}}(\mathbf{r})=\int \mathrm{d} \mathbf{r}^{\prime} v_{m c}\left(\left|\mathbf{r}-\mathbf{r}^{\prime}\right|\right) \rho_{m}\left(\mathbf{r}^{\prime}\right)
$$

the ensemble-averaged induced counterion density may be expanded in a functional Taylor series around $\phi_{\text {ext }}(\mathbf{r})=0$ [67] in powers of the dimensionless potential $u(\mathbf{r})=-\beta z e \phi_{\text {ext }}(\mathbf{r})$ :

$$
\left\langle\rho_{c}(\mathbf{r})\right\rangle=\rho_{0}+\sum_{n=1}^{\infty} \frac{1}{n !} \int \mathrm{d} \mathbf{r}_{1} \cdots \int \mathrm{d} \mathbf{r}_{n} G^{(n+1)}\left(\mathbf{r}-\mathbf{r}_{1}, \ldots, \mathbf{r}-\mathbf{r}_{n}\right) u\left(\mathbf{r}_{1}\right) \cdots u\left(\mathbf{r}_{n}\right) .
$$

Here $\rho_{0}$ is a constant, chosen below to ensure charge neutrality, and the coefficients

$$
G^{(n+1)}\left(\mathbf{r}-\mathbf{r}_{1}, \ldots, \mathbf{r}-\mathbf{r}_{n}\right)=\lim _{u \rightarrow 0}\left(\frac{\delta^{n}\left\langle\rho_{c}(\mathbf{r})\right\rangle}{\delta u\left(\mathbf{r}_{1}\right) \cdots \delta u\left(\mathbf{r}_{n}\right)}\right)
$$

are the $(n+1)$-particle density correlation functions [63] of the unperturbed (uniform) OCP. The correlation functions are, in turn, proportional to response functions (see below). To give a physical interpretation to Eq. (16), the counterion density induced at any point $\mathbf{r}$ is the net response to macroion-generated external potentials, applied at sets of points $\left\{\mathbf{r}_{1}, \ldots, \mathbf{r}_{n}\right\}$, and propagated through the OCP via multi-particle density correlations. Fourier transforming Eq. (16), we obtain (for $k \neq 0$ )

$$
\begin{aligned}
\left\langle\hat{\rho}_{c}(\mathbf{k})\right\rangle & =\hat{G}^{(2)}(k) \hat{u}(\mathbf{k})+\frac{1}{2 V^{\prime}} \sum_{\mathbf{k}^{\prime}} \hat{G}^{(3)}\left(\mathbf{k}^{\prime}, \mathbf{k}-\mathbf{k}^{\prime}\right) \hat{u}\left(\mathbf{k}^{\prime}\right) \hat{u}\left(\mathbf{k}-\mathbf{k}^{\prime}\right) \\
& +\frac{1}{3 ! V^{\prime 2}} \sum_{\mathbf{k}^{\prime}, \mathbf{k}^{\prime \prime}} \hat{G}^{(4)}\left(\mathbf{k}^{\prime}, \mathbf{k}^{\prime \prime}, \mathbf{k}-\mathbf{k}^{\prime}-\mathbf{k}^{\prime \prime}\right) \hat{u}\left(\mathbf{k}^{\prime}\right) \hat{u}\left(\mathbf{k}^{\prime \prime}\right) \hat{u}\left(\mathbf{k}-\mathbf{k}^{\prime}-\mathbf{k}^{\prime \prime}\right)+\cdots
\end{aligned}
$$

The coefficients $\hat{G}^{(n)}$, which are Fourier transforms of $G^{(n)}$, are related to the $n$-particle static structure factors of the uniform OCP via $\hat{G}^{(n)}=n_{c} S^{(n)}$, where the static structure factors are explicitly defined by 63

$$
S^{(2)}(k) \equiv S(k)=\frac{1}{N_{c}}\left\langle\hat{\rho}_{c}(\mathbf{k}) \hat{\rho}_{c}(-\mathbf{k})\right\rangle
$$


and

$$
S^{(n)}\left(\mathbf{k}_{1}, \cdots, \mathbf{k}_{n-1}\right)=\frac{1}{N_{c}}\left\langle\hat{\rho}_{c}\left(\mathbf{k}_{1}\right) \cdots \hat{\rho}_{c}\left(\mathbf{k}_{n-1}\right) \hat{\rho}_{c}\left(-\mathbf{k}_{1}-\ldots-\mathbf{k}_{n-1}\right)\right\rangle, \quad n \geq 3 .
$$

Substituting $\hat{u}(\mathbf{k})=-\beta \hat{v}_{m c}(k) \hat{\rho}_{m}(\mathbf{k})$ [from Eq. (15)] into Eq. (18), the induced counterion density can be expressed in the equivalent form

$$
\begin{aligned}
\left\langle\hat{\rho}_{c}(\mathbf{k})\right\rangle & =\chi(k) \hat{v}_{m c}(k) \hat{\rho}_{m}(\mathbf{k})+\frac{1}{V^{\prime}} \sum_{\mathbf{k}^{\prime}} \chi^{\prime}\left(\mathbf{k}^{\prime}, \mathbf{k}-\mathbf{k}^{\prime}\right) \hat{v}_{m c}\left(k^{\prime}\right) \hat{v}_{m c}\left(\left|\mathbf{k}-\mathbf{k}^{\prime}\right|\right) \\
& \times \hat{\rho}_{m}\left(\mathbf{k}^{\prime}\right) \hat{\rho}_{m}\left(\mathbf{k}-\mathbf{k}^{\prime}\right)+\cdots, \quad k \neq 0,
\end{aligned}
$$

where

$$
\chi(k)=-\beta n_{c} S(k)
$$

and

$$
\chi^{\prime}\left(\mathbf{k}^{\prime}, \mathbf{k}-\mathbf{k}^{\prime}\right)=\left(\beta^{2} n_{c} / 2\right) S^{(3)}\left(\mathbf{k}^{\prime}, \mathbf{k}-\mathbf{k}^{\prime}\right)
$$

are, respectively, the linear and the first nonlinear response function of the uniform OCP. The first term on the right side of Eq. (21) represents the linear response approximation - linear in $\hat{\rho}_{m}(\mathbf{k})$ - while the higher-order terms generate, as shown below, nonlinear corrections to both the counterion density and the effective interactions. Finally, since the amplitude of $\hat{v}_{m c}(k)$ is proportional to the macroion charge, then

$$
\begin{aligned}
\left\langle\hat{\rho}_{c}(\mathbf{k})\right\rangle_{\lambda} & =\lambda \chi(k) \hat{v}_{m c}(k) \hat{\rho}_{m}(\mathbf{k})+\frac{\lambda^{2}}{V^{\prime}} \sum_{\mathbf{k}^{\prime}} \chi^{\prime}\left(\mathbf{k}^{\prime}, \mathbf{k}-\mathbf{k}^{\prime}\right) \hat{v}_{m c}\left(k^{\prime}\right) \hat{v}_{m c}\left(\left|\mathbf{k}-\mathbf{k}^{\prime}\right|\right) \\
& \times \hat{\rho}_{m}\left(\mathbf{k}^{\prime}\right) \hat{\rho}_{m}\left(\mathbf{k}-\mathbf{k}^{\prime}\right)+\cdots, \quad k \neq 0 .
\end{aligned}
$$

Note that the response functions describe the response of the fully-charged OCP, and so do not depend on the coupling constant $\lambda$.

\section{Effective Interactions}

We are now positioned to derive formal expressions for the effective interactions. Substituting Eq. (24) into Eq. (14), the latter into Eq. (12), and integrating over $\lambda$, we obtain the counterion free energy to third order in the macroion density:

$$
\begin{gathered}
F_{c}=F_{\mathrm{OCP}}+n_{c} \lim _{k \rightarrow 0}\left[N_{m} \hat{v}_{m c}(k)+\frac{N_{c}}{2} \hat{v}_{c c}(k)\right]+\frac{1}{2 V^{\prime}} \sum_{\mathbf{k} \neq 0} \chi(k)\left[\hat{v}_{m c}(k)\right]^{2} \hat{\rho}_{m}(\mathbf{k}) \hat{\rho}_{m}(-\mathbf{k}) \\
+\frac{1}{3 V^{\prime 2}} \sum_{\mathbf{k} \neq 0} \sum_{\mathbf{k}^{\prime}} \chi^{\prime}\left(\mathbf{k}^{\prime},-\mathbf{k}-\mathbf{k}^{\prime}\right) \hat{v}_{m c}(k) \hat{v}_{m c}\left(k^{\prime}\right) \hat{v}_{m c}\left(\left|\mathbf{k}+\mathbf{k}^{\prime}\right|\right) \hat{\rho}_{m}(\mathbf{k}) \hat{\rho}_{m}\left(\mathbf{k}^{\prime}\right) \hat{\rho}_{m}\left(-\mathbf{k}-\mathbf{k}^{\prime}\right) .
\end{gathered}
$$

Evidently, the linear and first nonlinear response terms in the expansion of $\left\langle\hat{\rho}_{c}(\mathbf{k})\right\rangle$ generate terms in $F_{c}$ that are, respectively, quadratic and cubic in $\hat{\rho}_{m}(\mathbf{k})$. These terms can be related to effective pair and triplet interactions between macroions. To this end, we first identify

$$
\hat{v}_{\text {ind }}^{(2)}(k)=\chi(k)\left[\hat{v}_{m c}(k)\right]^{2}
$$

as the counterion-induced macroion-macroion pair interaction in the linear response approximation 32 , 33, 34]. In passing, we note that Eq. (26) is similar in structure and physical interpretation to induced interactions recently derived from a coarse-grained HNC theory [49] and from a cumulant expansion of the counterion partition function [68]. Combining the induced interaction with the bare Coulomb interaction yields the linear-response prediction for the total effective pair interaction:

$$
\hat{v}_{0}^{(2)}(k)=\hat{v}_{m m}(k)+\hat{v}_{\text {ind }}^{(2)}(k) .
$$


Now the term on the right side of Eq. (25) that is second-order in $\hat{\rho}_{m}(\mathbf{k})$ may be manipulated using the identity [from Eq. (5)]

$$
\sum_{i \neq j=1}^{N_{m}} v_{\text {ind }}^{(2)}\left(\left|\mathbf{R}_{i}-\mathbf{R}_{j}\right|\right)=\frac{1}{V^{\prime}} \sum_{\mathbf{k} \neq 0} \hat{v}_{\text {ind }}^{(2)}(k) \hat{\rho}_{m}(\mathbf{k}) \hat{\rho}_{m}(-\mathbf{k})+\frac{N_{m}^{2}}{V^{\prime}} \lim _{k \rightarrow 0} \hat{v}_{\text {ind }}^{(2)}(k)-N_{m} v_{\text {ind }}^{(2)}(0) .
$$

Similarly, identifying

$$
\hat{v}_{\text {eff }}^{(3)}\left(\mathbf{k}, \mathbf{k}^{\prime}\right)=2 \chi^{\prime}\left(\mathbf{k}^{\prime},-\mathbf{k}-\mathbf{k}^{\prime}\right) \hat{v}_{m c}(k) \hat{v}_{m c}\left(k^{\prime}\right) \hat{v}_{m c}\left(\left|\mathbf{k}+\mathbf{k}^{\prime}\right|\right)
$$

as an effective three-body interaction, arising from nonlinear counterion response, and using the identity

$$
\begin{aligned}
\sum_{i \neq j \neq k=1}^{N_{m}} v_{\mathrm{eff}}^{(3)}\left(\mathbf{R}_{i}-\mathbf{R}_{j}, \mathbf{R}_{i}-\mathbf{R}_{k}\right) & =\frac{1}{V^{\prime 2}} \sum_{\mathbf{k}} \sum_{\mathbf{k}^{\prime}} \hat{v}_{\mathrm{eff}}^{(3)}\left(\mathbf{k}, \mathbf{k}^{\prime}\right)\left[\hat{\rho}_{m}(\mathbf{k}) \hat{\rho}_{m}\left(\mathbf{k}^{\prime}\right) \hat{\rho}_{m}\left(-\mathbf{k}-\mathbf{k}^{\prime}\right)\right. \\
& \left.-3 \hat{\rho}_{m}(\mathbf{k}) \hat{\rho}_{m}(-\mathbf{k})+2 N_{m}\right],
\end{aligned}
$$

the final (third-order) term in Eq. (25) may be rewritten as

$$
\begin{aligned}
& \frac{1}{3 ! V^{\prime 2}} \sum_{\mathbf{k}} \sum_{\mathbf{k}^{\prime}} \hat{v}_{\mathrm{eff}}^{(3)}\left(\mathbf{k}, \mathbf{k}^{\prime}\right) \hat{\rho}_{m}(\mathbf{k}) \hat{\rho}_{m}\left(\mathbf{k}^{\prime}\right) \hat{\rho}_{m}\left(-\mathbf{k}-\mathbf{k}^{\prime}\right)-\frac{N_{m}}{3 ! V^{\prime 2}} \sum_{\mathbf{k}} \hat{v}_{\mathrm{eff}}^{(3)}(\mathbf{k}, 0) \hat{\rho}_{m}(\mathbf{k}) \hat{\rho}_{m}(-\mathbf{k}) \\
= & \frac{1}{3 !} \sum_{i \neq j \neq k}^{N_{m}} v_{\mathrm{eff}}^{(3)}\left(\mathbf{R}_{i}-\mathbf{R}_{j}, \mathbf{R}_{i}-\mathbf{R}_{k}\right)+\frac{1}{2 V^{\prime 2}} \sum_{\mathbf{k}} \sum_{\mathbf{k}^{\prime}} \hat{v}_{\mathrm{eff}}^{(3)}\left(\mathbf{k}, \mathbf{k}^{\prime}\right) \hat{\rho}_{m}(\mathbf{k}) \hat{\rho}_{m}(-\mathbf{k}) \\
- & \frac{N_{m}}{3 V^{\prime 2}} \sum_{\mathbf{k}} \sum_{\mathbf{k}^{\prime}} \hat{v}_{\mathrm{eff}}^{(3)}\left(\mathbf{k}, \mathbf{k}^{\prime}\right)-\frac{N_{m}}{3 ! V^{\prime 2}} \sum_{\mathbf{k}} \hat{v}_{\mathrm{eff}}^{(3)}(\mathbf{k}, 0) \hat{\rho}_{m}(\mathbf{k}) \hat{\rho}_{m}(-\mathbf{k}) .
\end{aligned}
$$

Combining Eqs. (25) and (31), and again invoking the identity in Eq. (5), the effective Hamiltonian acquires the following physically intuitive structure:

$$
H_{\mathrm{eff}}=H_{\mathrm{HS}}+\frac{1}{2} \sum_{i \neq j=1}^{N_{m}} v_{\mathrm{eff}}^{(2)}\left(\left|\mathbf{R}_{i}-\mathbf{R}_{j}\right|\right)+\frac{1}{3 !} \sum_{i \neq j \neq k=1}^{N_{m}} v_{\mathrm{eff}}^{(3)}\left(\mathbf{R}_{i}-\mathbf{R}_{j}, \mathbf{R}_{i}-\mathbf{R}_{k}\right)+E
$$

where $v_{\text {eff }}^{(2)}(r)$ and $v_{\text {eff }}^{(3)}\left(\mathbf{r}, \mathbf{r}^{\prime}\right)$ are, respectively, the counterion-induced effective pair and triplet interactions in real space and $E$ is a one-body volume energy. In Eq. (32), the effective triplet interaction is the Fourier transform of Eq. (29), while the effective pair interaction is the transform of

$$
\hat{v}_{\mathrm{eff}}^{(2)}(k)=\hat{v}_{0}^{(2)}(k)+\Delta \hat{v}_{\mathrm{eff}}^{(2)}(k),
$$

where

$$
\Delta \hat{v}_{\mathrm{eff}}^{(2)}(k)=\frac{1}{V^{\prime}} \sum_{\mathbf{k}^{\prime}} \hat{v}_{\mathrm{eff}}^{(3)}\left(\mathbf{k}, \mathbf{k}^{\prime}\right)-\frac{N_{m}}{3 V^{\prime}} \hat{v}_{\mathrm{eff}}^{(3)}(\mathbf{k}, 0)
$$

is the first nonlinear correction to the linear response approximation. Note that the second term on the right side of Eq. (34) can be traced back to the requirement of charge neutrality, which necessitated special treatment of the $k=0$ term in Eq. (13).

The volume energy $E$ - a natural by-product of reduction to an equivalent one-component system has no explicit dependence on macroion coordinates. Collecting terms that are independent of macroion coordinates, the volume energy takes the form

$$
E=E_{0}+\Delta E,
$$

where

$$
E_{0}=F_{\mathrm{OCP}}+\frac{N_{m}}{2} v_{\text {ind }}^{(2)}(0)+N_{m} n_{c} \lim _{k \rightarrow 0}\left[\hat{v}_{m c}(k)-\frac{z}{2 Z} \hat{v}_{\text {ind }}^{(2)}(k)+\frac{Z}{2 z} \hat{v}_{c c}(k)\right]
$$


is the linear response approximation [33, 34] and

$$
\Delta E=\frac{N_{m}}{6 V^{\prime 2}}\left[\sum_{\mathbf{k}, \mathbf{k}^{\prime}} \hat{v}_{\mathrm{eff}}^{(3)}\left(\mathbf{k}, \mathbf{k}^{\prime}\right)-N_{m} \sum_{\mathbf{k}} \hat{v}_{\mathrm{eff}}^{(3)}(\mathbf{k}, 0)\right]
$$

is the first nonlinear correction. On the right side of Eq. (36), the second term represents the interaction of a macroion with its own counterions. The terms in square brackets on the right side of Eq. (36) and the second term on the right side of Eq. (37) originate again from the requirement of charge neutrality. We emphasize that nonlinear counterion response generates not only effective many-body interactions, but also corrections to both the effective pair interaction and the volume energy. In fact, as is clear from Eqs. (34) and (37), the nonlinear corrections to $v_{\text {eff }}^{(2)}(r)$ and $E$ are intimately related to many-body interactions. Note that the volume energy depends nontrivially on the mean macroion density, and thus can contribute significantly to the total free energy of the system.

\section{Physical Interpretation}

While the mathematical manipulations of response theory are simpler in Fourier space, the physical interpretation of the theory is perhaps more transparent in real space. In terms of real-space functions, the induced pair interaction, in the linear response approximation, can be expressed [from Eq. (26)] as

$$
v_{\text {ind }}^{(2)}(r)=\int \mathrm{d} \mathbf{r}_{1} \int \mathrm{d} \mathbf{r}_{2} \chi\left(\left|\mathbf{r}_{1}-\mathbf{r}_{2}\right|\right) v_{m c}\left(r_{1}\right) v_{m c}\left(\left|\mathbf{r}_{2}-\mathbf{r}\right|\right),
$$

where $\chi\left(\left|\mathbf{r}_{1}-\mathbf{r}_{2}\right|\right)$ is the real-space linear response function, which describes the change in counterion density induced at point $\mathbf{r}_{2}$ in response to an external potential applied at $\mathbf{r}_{1}$. Referring to Fig. 20 Eq. (38) can be interpreted as follows. The external potential due to one macroion (centered at the origin in Fig. 2) induces at point $\mathbf{r}_{2}$ a change in counterion density $\int \mathrm{d} \mathbf{r}_{1} \chi\left(\left|\mathbf{r}_{1}-\mathbf{r}_{2}\right|\right) v_{m c}\left(r_{1}\right)$. This induced density, which depends (via $\chi$ ) on the pair density correlation function of the intervening medium (OCP), then interacts with a second macroion, at displacement $\mathbf{r}$ from the first, giving rise to a counterion-induced pair interaction energy. The linear-response contribution to the volume energy (per macroion) associated with macroioncounterion interactions [Eq. (36)] has a closely related form

$$
v_{\text {ind }}^{(2)}(0)=\int \mathrm{d} \mathbf{r}_{1} \int \mathrm{d} \mathbf{r}_{2} \chi\left(\left|\mathbf{r}_{1}-\mathbf{r}_{2}\right|\right) v_{m c}\left(r_{1}\right) v_{m c}\left(r_{2}\right)
$$

and a similar physical interpretation, except that the induced density now interacts back with the first macroion, generating a one-body energy.

Proceeding from linear to nonlinear response, the effective triplet interaction can be expressed [from Eq. (29)] as

$$
v_{\text {eff }}^{(3)}\left(\mathbf{r}, \mathbf{r}^{\prime}\right)=2 \int \mathrm{d} \mathbf{r}_{1} \int \mathrm{d} \mathbf{r}_{2} \int \mathrm{d} \mathbf{r}_{3} \chi^{\prime}\left(\mathbf{r}_{1}-\mathbf{r}_{3}, \mathbf{r}_{2}-\mathbf{r}_{3}\right) v_{m c}\left(r_{1}\right) v_{m c}\left(\left|\mathbf{r}_{\mathbf{2}}-\mathbf{r}\right|\right) v_{m c}\left(\left|\mathbf{r}_{\mathbf{3}}-\mathbf{r}^{\prime}\right|\right) .
$$

Again the interpretation is clear: the external potentials due to two macroions (top two macroions in Fig. 2), separated by displacement $\mathbf{r}$, induce a change in counterion density at point $\mathbf{r}_{3}$. The induced density, which depends via $\chi^{\prime}$ on triplet density correlations in the OCP, then interacts with a third macroion, at displacement $\mathbf{r}^{\prime}$ from the first, contributing a counterion-induced three-particle interaction energy. Considering now the nonlinear correction to the pair interaction, and leaving aside the term arising from charge neutrality, the main contribution can be written [from Eq. (34)] as

$$
\Delta v_{\text {eff }}^{(2)}(r)=2 \int \mathrm{d} \mathbf{r}_{1} \int \mathrm{d} \mathbf{r}_{2} \int \mathrm{d} \mathbf{r}_{3} \chi^{\prime}\left(\mathbf{r}_{1}-\mathbf{r}_{3}, \mathbf{r}_{2}-\mathbf{r}_{3}\right) v_{m c}\left(r_{1}\right) v_{m c}\left(r_{2}\right) v_{m c}\left(\left|\mathbf{r}_{\mathbf{3}}-\mathbf{r}\right|\right) .
$$

The interpretation is analogous to that for the triplet interaction, except that the external potentials at points $\mathbf{r}_{1}$ and $\mathbf{r}_{2}$ are now associated with the same macroion. Finally, the nonlinear correction to the volume 
energy [Eq. [37)], aside from the charge neutrality term, has the form

$$
\Delta E=\frac{N_{m}}{3} \int \mathrm{d} \mathbf{r}_{1} \int \mathrm{d} \mathbf{r}_{2} \int \mathrm{d} \mathbf{r}_{3} \chi^{\prime}\left(\mathbf{r}_{1}-\mathbf{r}_{3}, \mathbf{r}_{2}-\mathbf{r}_{3}\right) v_{m c}\left(r_{1}\right) v_{m c}\left(r_{2}\right) v_{m c}\left(r_{3}\right)
$$

The physical meaning of $\Delta E$ is similar to that of $\Delta v_{\mathrm{eff}}^{(2)}(r)$, except that now the density that is induced nonlinearly by one macroion interacts back with the same macroion, generating a nonlinear contribution to the one-body energy.

\section{E. Random Phase Approximation}

Further progress towards practical expressions for effective interactions requires specifying the OCP response functions. For charged colloids, the OCP is typically weakly correlated, characterized by relatively small coupling parameters: $\Gamma=\lambda_{B} / a_{c} \ll 1$, where $\lambda_{B}=\beta z^{2} e^{2} / \epsilon$ is the Bjerrum length and $a_{c}=\left(3 / 4 \pi n_{c}\right)^{1 / 3}$ is the counterion-sphere radius. For example, for macroions of valence $Z=500$, volume fraction $\eta=0.01$, and monovalent counterions suspended in salt-free water at room temperature $\left(\lambda_{B}=0.714 \mathrm{~nm}\right)$, we find $\Gamma \simeq 0.02$. For such weakly-correlated plasmas, it is reasonable - at least as regards long-range interactions - to neglect short-range correlations. We thus adopt the random phase approximation (RPA), which equates the two-particle direct correlation function (DCF) to its exact asymptotic limit: $c^{(2)}(r)=-\beta v_{c c}(r)$ or $\hat{c}^{(2)}(k)=-4 \pi \beta z^{2} e^{2} / \epsilon k^{2}$. In neglecting short-range correlations, the RPA is formally equivalent to the mean-field PB theory, as shown in the Appendix. Furthermore, we ignore the influence of the macroion hard cores on the OCP response functions, which is reasonable for sufficiently dilute suspensions. Within the RPA, the OCP (two-particle) static structure factor and linear response function take the analytical forms

$$
S(k)=\frac{1}{1-n_{c} \hat{c}^{(2)}(k)}=\frac{1}{1+\kappa^{2} / k^{2}}
$$

and

$$
\chi(k)=-\beta n_{c} S(k)=\frac{-\beta n_{c}}{1+\kappa^{2} / k^{2}},
$$

where $\kappa=\sqrt{4 \pi n_{c} z^{2} e^{2} / \epsilon k_{B} T}$. As will be seen below, the parameter $\kappa$ plays the role of the Debye screening constant (inverse screening length) in the counterion density profile and in the effective interactions. In the absence of salt, the counterions are the only screening ions. The macroions themselves, being singled out as sources of the external potential for the counterions, do not contribute to the density of screening ions. Fourier transforming Eq. (44), the real-space linear response function takes the form

$$
\chi(r)=-\beta n_{c}\left[\delta(\mathbf{r})+n_{c} h_{c c}(r)\right],
$$

where

$$
h_{c c}(r)=-\frac{\beta z^{2} e^{2}}{\epsilon} \frac{e^{-\kappa r}}{r}
$$

is the counterion-counterion pair correlation function [69], which has Yukawa form, with screening constant $\kappa$. Equation (45) makes clear that there are two physically distinct types of counterion response: local response, associated with counterion self correlations, and nonlocal response, associated with counterion pair correlations.

At this point, we can specify the constant $\rho_{0}$ in Eq. (16). Combining Eq. (44) with the long-wavelength limit of the macroion-counterion interaction, $\hat{v}_{m c}(k) \rightarrow 4 \pi Z z e^{2} / \epsilon k^{2}$, as $k \rightarrow 0$, we have

$$
\lim _{k \rightarrow 0}\left[\chi(k) \hat{v}_{m c}(k)\right]=Z / z .
$$

Thus, the linear response term in Eq. (16) already ensures proper normalization of $\rho_{c}(\mathbf{r})$, which implies that $\rho_{0}=0$. 
Proceeding to nonlinear response, we first note that the three-particle structure factor obeys the identity

$$
S^{(3)}\left(\mathbf{k}, \mathbf{k}^{\prime}\right)=S(k) S\left(k^{\prime}\right) S\left(\left|\mathbf{k}+\mathbf{k}^{\prime}\right|\right)\left[1+n_{c}^{2} \hat{c}^{(3)}\left(\mathbf{k}, \mathbf{k}^{\prime}\right)\right]
$$

where $\hat{c}^{(3)}\left(\mathbf{k}, \mathbf{k}^{\prime}\right)$ is the Fourier transform of the three-particle direct correlation function. Within the RPA, however, $c^{(3)}$ and all higher-order DCF's vanish. Thus, from Eqs. (22), (23), and (48), the first nonlinear response function can be expressed in Fourier space as

$$
\chi^{\prime}\left(\mathbf{k}, \mathbf{k}^{\prime}\right)=-\frac{k_{\mathrm{B}} T}{2 n_{c}^{2}} \chi(k) \chi\left(k^{\prime}\right) \chi\left(\left|\mathbf{k}+\mathbf{k}^{\prime}\right|\right)
$$

and in real space as

$$
\chi^{\prime}\left(\mathbf{r}_{1}-\mathbf{r}_{2}, \mathbf{r}_{1}-\mathbf{r}_{3}\right)=-\frac{k_{\mathrm{B}} T}{2 n_{c}^{2}} \int \mathrm{d} \mathbf{r} \chi\left(\left|\mathbf{r}_{1}-\mathbf{r}\right|\right) \chi\left(\left|\mathbf{r}_{2}-\mathbf{r}\right|\right) \chi\left(\left|\mathbf{r}_{3}-\mathbf{r}\right|\right) .
$$

Higher-order nonlinear counterion response leads to higher-order terms in the effective Hamiltonian [Eq. (32)]. For example, the effective four-body interaction takes the form

$$
v_{\mathrm{eff}}^{(4)}\left(\mathbf{k}, \mathbf{k}^{\prime}, \mathbf{k}^{\prime \prime}\right)=6 \chi^{\prime \prime}\left(\mathbf{k}^{\prime}, \mathbf{k}^{\prime \prime},-\mathbf{k}-\mathbf{k}^{\prime}-\mathbf{k}^{\prime \prime}\right) \hat{v}_{m c}(k) \hat{v}_{m c}\left(k^{\prime}\right) \hat{v}_{m c}\left(k^{\prime \prime}\right) \hat{v}_{m c}\left(\left|\mathbf{k}+\mathbf{k}^{\prime}+\mathbf{k}^{\prime \prime}\right|\right),
$$

where

$$
\chi^{\prime \prime}\left(\mathbf{k}, \mathbf{k}^{\prime}, \mathbf{k}^{\prime \prime}\right)=\frac{-\beta^{3}}{3 !} n_{c} S^{(4)}\left(\mathbf{k}, \mathbf{k}^{\prime}, \mathbf{k}^{\prime \prime}\right)
$$

is the next higher-order nonlinear response function and

$$
\begin{aligned}
S^{(4)}\left(\mathbf{k}, \mathbf{k}^{\prime}, \mathbf{k}^{\prime \prime}\right) & =S(k) S\left(k^{\prime}\right) S\left(k^{\prime \prime}\right) S\left(\left|\mathbf{k}+\mathbf{k}^{\prime}+\mathbf{k}^{\prime \prime}\right|\right) \\
& \times\left[S\left(\left|\mathbf{k}+\mathbf{k}^{\prime}\right|\right)+S\left(\left|\mathbf{k}^{\prime}+\mathbf{k}^{\prime \prime}\right|\right)+S\left(\left|\mathbf{k}+\mathbf{k}^{\prime \prime}\right|\right)-2\right]
\end{aligned}
$$

is the four-particle structure factor in the RPA. Just as effective three-body interactions are related to corrections at the two- and one-body levels, so four-body interactions entail corrections at the three-, two-, and one-body levels, which (in Fourier space) are proportional to appropriate summations of $v_{\mathrm{eff}}^{(4)}\left(\mathbf{k}, \mathbf{k}^{\prime}, \mathbf{k}^{\prime \prime}\right)$ over the wave-vectors $\mathbf{k}, \mathbf{k}^{\prime}$, and $\mathbf{k}^{\prime \prime}$. In principle, these higher-order corrections could be computed to further check for convergence of the perturbation expansion.

\section{RESULTS}

\section{A. Counterion Density}

A practical expression for the ensemble-averaged counterion density is now obtained by substituting the linear and first nonlinear RPA response functions [Eqs. (44) and (49)] into the expansion for $\left\langle\hat{\rho}_{c}(\mathbf{k})\right\rangle[\mathrm{Eq}$. (21)]. The result may be expressed in the form

$$
\left\langle\hat{\rho}_{c}(\mathbf{k})\right\rangle=\hat{\rho}_{c 0}(\mathbf{k})-\frac{\chi(k)}{2 \beta n_{c}^{2} V^{\prime}} \sum_{\mathbf{k}^{\prime}} \hat{\rho}_{c 0}\left(\mathbf{k}^{\prime}\right) \hat{\rho}_{c 0}\left(\mathbf{k}-\mathbf{k}^{\prime}\right), \quad k \neq 0,
$$

where

$$
\hat{\rho}_{c 0}(\mathbf{k})=\chi(k) \hat{v}_{m c}(k) \hat{\rho}_{m}(\mathbf{k}), \quad k \neq 0,
$$

is the Fourier transform of the linear-response counterion density and $\hat{v}_{m c}(k)$ is the transform of the macroioncounterion interaction (specified below). Inverse transforming Eq. (54) yields

$$
\left\langle\rho_{c}(\mathbf{r})\right\rangle=\rho_{c 0}(\mathbf{r})-\frac{1}{2 \beta n_{c}^{2}} \int \mathrm{d} \mathbf{r}^{\prime} \chi\left(\left|\mathbf{r}-\mathbf{r}^{\prime}\right|\right)\left[\rho_{c 0}\left(\mathbf{r}^{\prime}\right)\right]^{2}
$$


where

$$
\rho_{c 0}(\mathbf{r})=\sum_{i=1}^{N_{m}} \rho_{1}\left(\left|\mathbf{r}-\mathbf{R}_{i}\right|\right)
$$

is the real-space linear response counterion density in the presence of macroions fixed at positions $\mathbf{R}_{i}$, expressed as a sum of single-macroion counterion density orbitals $\rho_{1}(r)$ - the inverse transform of $\hat{\rho}_{1}(k)=$ $\chi(k) \hat{v}_{m c}(k)$. Equivalently,

$$
\rho_{c 0}(\mathbf{r})=\int \mathrm{d} \mathbf{r}^{\prime} \chi\left(\left|\mathbf{r}-\mathbf{r}^{\prime}\right|\right) \sum_{i=1}^{N_{m}} v_{m c}\left(\left|\mathbf{r}^{\prime}-\mathbf{R}_{i}\right|\right) .
$$

Now substitution of Eqs. (45) and (46) for the real-space RPA linear response function into Eqs. (56) and (58) allows the linear-response counterion density profile to be expressed as

$$
\rho_{c 0}(\mathbf{r})=\beta n_{c} \sum_{i=1}^{N_{m}}\left[-v_{m c}\left(\left|\mathbf{r}-\mathbf{R}_{i}\right|\right)+\frac{\kappa^{2}}{4 \pi} \int \mathrm{d} \mathbf{r}^{\prime} \frac{e^{-\kappa\left|\mathbf{r}-\mathbf{r}^{\prime}\right|}}{\left|\mathbf{r}-\mathbf{r}^{\prime}\right|} v_{m c}\left(\left|\mathbf{r}^{\prime}-\mathbf{R}_{i}\right|\right)\right]
$$

and the nonlinear profile as

$$
\left\langle\rho_{c}(\mathbf{r})\right\rangle=\rho_{c 0}(\mathbf{r})+\frac{1}{2 n_{c}}\left[\rho_{c 0}(\mathbf{r})\right]^{2}-\frac{\kappa^{2}}{8 \pi n_{c}} \int \mathrm{d} \mathbf{r}^{\prime} \frac{e^{-\kappa\left|\mathbf{r}-\mathbf{r}^{\prime}\right|}}{\left|\mathbf{r}-\mathbf{r}^{\prime}\right|}\left[\rho_{c 0}\left(\mathbf{r}^{\prime}\right)\right]^{2} .
$$

The last two terms on the right side of Eq. (60) are nonlinear corrections to the linear profile and can be physically interpreted as arising, respectively, from local and nonlocal nonlinear response of counterions to the macroion charge.

The linear-response counterion profile in the presence of a distribution of macroions can be obtained by ensemble averaging Eq. (55) over macroion coordinates. In Fourier space,

$$
\left\langle\hat{\rho}_{c 0}(\mathbf{k})\right\rangle_{m}=\rho_{1}(k)\langle\hat{\rho}(\mathbf{k})\rangle_{m}
$$

where \langle\rangle$_{m}$ again represents a trace over macroion coordinates and $\langle\hat{\rho}(\mathbf{k})\rangle_{m}$ is the Fourier component of the average density of macroions. In real space, the average density of counterions around a central macroion can then be expressed as

$$
\left\langle\rho_{c 0}(\mathbf{r})\right\rangle_{m}=\rho_{1}(\mathbf{r})+n_{m} \int \mathrm{d} \mathbf{r}^{\prime} g_{m m}\left(\mathbf{r}^{\prime}\right) \rho_{1}\left(\left|\mathbf{r}-\mathbf{r}^{\prime}\right|\right),
$$

where $g_{m m}(\mathbf{r})$ is the macroion-macroion pair distribution function. The latter function may be obtained from integral-equation theory or simulation, with effective interactions as input.

\section{B. Macroion-Counterion Interaction}

To this point, the theory makes no assumptions about the type of macroion. Practical calculations require specifying the macroion structure, the macroion-counterion interaction, and the corresponding singlemacroion counterion density orbital. Henceforth, we specialize to charged hard-sphere colloidal macroions. A convenient strategy, proposed in ref. [30] and adopted in refs. [33] and [34], specifies $v_{m c}(r)$ inside the hard core $(r<a)$ so as to minimize counterion penetration. We thus assume

$$
v_{m c}(r)= \begin{cases}\frac{-Z z e^{2}}{\epsilon r}, & r>a \\ \frac{-Z z e^{2}}{\epsilon a} \alpha, & r<a\end{cases}
$$

and choose the parameter $\alpha$ appropriately. In passing, we note that $v_{m c}(r)$ plays a role here analogous to that of an empty-core pseudopotential in the pseudopotential theory of simple metals [65, 66]. As shown 
in refs. [30] and [33], at the level of linear response, penetration of counterions inside the macroion cores is eliminated by choosing $\alpha=\kappa a /(1+\kappa a)$. This choice yields

$$
\hat{v}_{\mathrm{mc}}(k)=-\frac{4 \pi Z z e^{2}}{\epsilon(1+\kappa a) k^{2}}\left[\cos (k a)+\frac{\kappa}{k} \sin (k a)\right]
$$

and

$$
\rho_{1}(r)= \begin{cases}\frac{Z}{z} \frac{\kappa^{2}}{4 \pi} \frac{e^{\kappa a}}{1+\kappa a} \frac{e^{-\kappa r}}{r}, & r>a \\ 0, & r<a\end{cases}
$$

which is precisely the DLVO expression for the density of counterions around an isolated macroion [27, 28]. The above choice for the parameter $\alpha$ ensures that the linear term and first nonlinear term of Eq. (60) vanish completely inside the macroion core. The same parametrization also allows, however, the final nonlinear term in Eq. (60) to be nonzero inside the core, although in practice the fractional penetration is at most a few percent. Independent of parametrization, Eqs. (56), (57), and (60) maintain charge neutrality by preserving the number of counterions, since $\int \mathrm{d} \mathbf{r} \rho_{1}(\mathbf{r})=Z / z$ and $\int \mathrm{d} \mathbf{r} \chi(r)=\chi(k=0)=0$.

Another artifact of the present scheme, apparent from Eq. (57), is that the counterion density profile around a given macroion overlaps the hard cores of neighboring macroions. More general parametrizations of the macroion-counterion interaction than Eq. (63) could conceivably eliminate counterion penetration within all cores. An alternative strategy would incorporate excluded volume constraints directly into the response functions, which then would more properly describe the Swiss cheese OCP. In such a scheme, $\chi\left(\left|\mathbf{r}-\mathbf{r}^{\prime}\right|\right)$ would strictly vanish when either $\mathbf{r}$ or $\mathbf{r}^{\prime}$ falls inside a hard core. This condition - not obeyed by Eqs. (44) and (49) - would enforce exclusion of counterions from all macroion cores. Nevertheless, in the current scheme, the extent of core overlap is minor for macroion separations that significantly exceed the screening length $\kappa^{-1}$, which is usually the case in practice.

\section{Effective Interactions}

The effective interactions can be expressed in real space by evaluating the respective inverse Fourier transforms. From Eqs. (29) and (49), the effective triplet interaction is

$$
v_{\mathrm{eff}}^{(3)}\left(\mathbf{r}_{1}-\mathbf{r}_{2}, \mathbf{r}_{1}-\mathbf{r}_{3}\right)=-\frac{k_{B} T}{n_{c}^{2}} \int \mathrm{d} \mathbf{r} \rho_{1}\left(\left|\mathbf{r}_{1}-\mathbf{r}\right|\right) \rho_{1}\left(\left|\mathbf{r}_{2}-\mathbf{r}\right|\right) \rho_{1}\left(\left|\mathbf{r}_{3}-\mathbf{r}\right|\right)
$$

Equations (26) and (27), combined with Eqs. (44) and (64), yield the linear-response prediction for the effective pair interaction [33],

$$
v_{0}^{(2)}(r)=\frac{Z^{2} e^{2}}{\epsilon}\left(\frac{e^{\kappa a}}{1+\kappa a}\right)^{2} \frac{e^{-\kappa r}}{r}, \quad r>\sigma,
$$

identical to the familiar DLVO screened-Coulomb potential in the limit of widely separated macroions [27, 28], while Eq. (34) yields the first nonlinear correction

$$
\Delta v_{\mathrm{eff}}^{(2)}(r)=-\frac{k_{B} T}{n_{c}^{2}} \int \mathrm{d} \mathbf{r}^{\prime} \rho_{1}\left(r^{\prime}\right) \rho_{1}\left(\left|\mathbf{r}-\mathbf{r}^{\prime}\right|\right)\left[\rho_{1}\left(\left|\mathbf{r}-\mathbf{r}^{\prime}\right|\right)-\frac{n_{c}}{3}\right] .
$$

The total effective pair potential is given by $v_{\mathrm{eff}}^{(2)}(r)=v_{0}^{(2)}(r)+\Delta v_{\mathrm{eff}}^{(2)}(r)$. Note the distinction between the effective pair potential, which is the interaction between a pair of macroions in a colloidal suspension of arbitrary concentration, and the potential of mean force, which is the interaction between an isolated pair of macroions, i.e., the low-density limit of $v_{\mathrm{eff}}^{(2)}(r)$.

The volume energy can be expressed - by combining Eqs. (26), (29), (36), (37), and (64) - as the sum of the linear response prediction 33],

$$
E_{0}=F_{\mathrm{OCP}}-N_{m} \frac{Z^{2} e^{2}}{2 \epsilon} \frac{\kappa}{1+\kappa a}-\frac{N_{c} k_{B} T}{2},
$$


and the first nonlinear correction,

$$
\Delta E=-\frac{N_{m} k_{B} T}{6 n_{c}^{2}}\left(\int \mathrm{d} \mathbf{r}\left[\rho_{1}(r)\right]^{3}-n_{c} \int \mathrm{d} \mathbf{r}\left[\rho_{1}(r)\right]^{2}\right) .
$$

The first and second terms on the right side of Eq. (69) account, respectively, for the counterion entropy and the macroion-counterion electrostatic interaction energy, while Eq. (70) corrects the latter term for nonlinear response. The final terms on the right sides of Eqs. (68)-(70) originate from the charge neutrality constraint.

\section{Effect of Added Salt}

Finally, we generalize the above results to the case of nonzero salt concentration. The average number density (in the free volume) of salt ion pairs, $n_{s}=N_{s} / V^{\prime}$, is supposed maintained by exchange of salt ions with a salt reservoir through a semi-permeable membrane. The total average microion density is then $n_{\mu}=n_{+}+n_{-}=n_{c}+2 n_{s}$, where $n_{ \pm}$are the average number densities of positive/negative microions. Following ref. [34], the Hamiltonian generalizes to

$$
H=H_{m}+H_{\mu}+H_{m+}+H_{m-},
$$

where $H_{\mu}$ is the Hamiltonian of the microions (counterions plus salt ions) and $H_{m \pm}$ are the electrostatic interaction energies between macroions and positive/negative microions. The presence of positive and negative microion species requires a proliferation of response functions, $\chi_{i j}$ and $\chi_{i j k}^{\prime}, i, j, k= \pm$, and a generalization of Eq. (45) to

$$
\begin{gathered}
\chi_{++}(r)=-\beta n_{+}\left[\delta(\mathbf{r})+n_{+} h_{++}(r)\right], \\
\chi_{+-}(r)=-\beta n_{+} n_{-} h_{+-}(r), \\
\chi_{--}(r)=-\beta n_{-}\left[\delta(\mathbf{r})+n_{-} h_{--}(r)\right],
\end{gathered}
$$

where $h_{i j}(r), i, j= \pm$, are the bulk microion two-particle pair correlation functions, which depend implicitly on $n_{ \pm}$. Generalizing Eq. (21), the ensemble-averaged microion number densities are given by

$$
\begin{aligned}
\left\langle\hat{\rho}_{ \pm}(\mathbf{k})\right\rangle & = \pm \chi_{ \pm}(k) \hat{v}_{m+}(k) \hat{\rho}_{m}(\mathbf{k})+\frac{1}{V^{\prime}} \sum_{\mathbf{k}^{\prime}} \chi_{ \pm}^{\prime}\left(\mathbf{k}^{\prime}, \mathbf{k}-\mathbf{k}^{\prime}\right) \hat{v}_{m+}\left(k^{\prime}\right) \hat{v}_{m+}\left(\left|\mathbf{k}-\mathbf{k}^{\prime}\right|\right) \\
& \times \hat{\rho}_{m}\left(\mathbf{k}^{\prime}\right) \hat{\rho}_{m}\left(\mathbf{k}-\mathbf{k}^{\prime}\right)+\cdots \quad(k \neq 0)
\end{aligned}
$$

where we have exploited symmetries: $\hat{v}_{m+}=-\hat{v}_{m-}, \chi_{+-}=\chi_{-+}, \chi_{++-}^{\prime}=\chi_{+-+}^{\prime}$, etc., to define composite response functions as $\chi_{+}=\chi_{++}-\chi_{+-}, \chi_{-}=\chi_{--}-\chi_{+-}, \chi_{+}^{\prime}=\chi_{+++}^{\prime}-2 \chi_{++-}^{\prime}+\chi_{+--}^{\prime}$, and $\chi_{-}^{\prime}=\chi_{---}^{\prime}$ $2 \chi_{-+-}^{\prime}+\chi_{-++}^{\prime}$. Substituting Eq. (75) into the multi-component Hamiltonian [Eq. (71)], the macroionmicroion interaction contribution can be expressed as

$$
\begin{aligned}
H_{m+}+H_{m-} & =\frac{1}{V^{\prime}} \sum_{\mathbf{k}} \chi(k)\left[\hat{v}_{m+}(k)\right]^{2} \hat{\rho}_{m}(\mathbf{k}) \hat{\rho}_{m}(-\mathbf{k})+\frac{1}{V^{\prime 2}} \sum_{\mathbf{k}, \mathbf{k}^{\prime}} \chi^{\prime}\left(\mathbf{k}^{\prime},-\mathbf{k}-\mathbf{k}^{\prime}\right) \\
& \times \hat{v}_{m+}(k) \hat{v}_{m+}\left(k^{\prime}\right) \hat{v}_{m+}\left(\left|\mathbf{k}+\mathbf{k}^{\prime}\right|\right) \hat{\rho}_{m}(\mathbf{k}) \hat{\rho}_{m}\left(\mathbf{k}^{\prime}\right) \hat{\rho}_{m}\left(-\mathbf{k}-\mathbf{k}^{\prime}\right),
\end{aligned}
$$

where the linear and first nonlinear response functions are now redefined as the following combinations of $\chi_{i j}$ and $\chi_{i j k}^{\prime}$ :

$$
\chi=\chi_{+}+\chi_{-}=\chi_{++}-2 \chi_{+-}+\chi_{--}
$$

and

$$
\chi^{\prime}=\chi_{+}^{\prime}-\chi_{-}^{\prime}=\chi_{+++}^{\prime}-3 \chi_{++-}^{\prime}+3 \chi_{+--}^{\prime}-\chi_{---}^{\prime} .
$$


The net effect of adding salt is to modify the previous salt-free results as follows. First, the average counterion density $n_{c}$ in the Debye screening constant $\kappa$ and in the linear response function [Eq. (44)] must be replaced by the total average microion density $n_{\mu}$. Thus, $\kappa \rightarrow \sqrt{4 \pi n_{\mu} z^{2} e^{2} / \epsilon k_{B} T}$ and $\chi(k) \rightarrow-\beta n_{\mu} S(k)$. The first nonlinear response function retains its original form [Eq. (49)], but with the new definition of $\kappa$. The second modification is in the linear-response volume energy [Eq. [69]), which becomes [34, 70]

$$
E_{0}=F_{\text {plasma }}-N_{m} \frac{Z^{2} e^{2}}{2 \epsilon} \frac{\kappa}{1+\kappa a}-\frac{k_{B} T}{2} \frac{\left(N_{+}-N_{-}\right)^{2}}{N_{+}+N_{-}},
$$

where $F_{\text {plasma }}$ is the free energy of the unperturbed microion plasma. Finally, the effective triplet interaction and nonlinear corrections to the effective pair interaction and volume energy are generalized as follows:

$$
\begin{gathered}
\Delta E=-\frac{N_{m} k_{B} T}{6} \frac{\left(n_{+}-n_{-}\right)}{n_{\mu}^{3}}\left(\int \mathrm{d} \mathbf{r}\left[\rho_{1}(r)\right]^{3}-n_{\mu} \int \mathrm{d} \mathbf{r}\left[\rho_{1}(r)\right]^{2}\right) \\
\Delta v_{\mathrm{eff}}^{(2)}(r)=-k_{B} T \frac{\left(n_{+}-n_{-}\right)}{n_{\mu}^{3}} \int \mathrm{d} \mathbf{r}^{\prime} \rho_{1}\left(r^{\prime}\right) \rho_{1}\left(\left|\mathbf{r}-\mathbf{r}^{\prime}\right|\right)\left[\rho_{1}\left(\left|\mathbf{r}-\mathbf{r}^{\prime}\right|\right)-\frac{n_{\mu}}{3}\right], \\
v_{\mathrm{eff}}^{(3)}\left(\mathbf{r}_{1}-\mathbf{r}_{2}, \mathbf{r}_{1}-\mathbf{r}_{3}\right)=-k_{B} T \frac{\left(n_{+}-n_{-}\right)}{n_{\mu}^{3}} \int \mathrm{d} \mathbf{r} \rho_{1}\left(\left|\mathbf{r}_{1}-\mathbf{r}\right|\right) \rho_{1}\left(\left|\mathbf{r}_{2}-\mathbf{r}\right|\right) \rho_{1}\left(\left|\mathbf{r}_{3}-\mathbf{r}\right|\right) .
\end{gathered}
$$

Equations (80)- (82) [combined with Eq. (65) for $\rho_{1}(r)$ ] are the main new results for nonlinear effective interactions. These expressions imply that nonlinear effects increase in strength with increasing macroion charge, increasing macroion concentration, and decreasing salt concentration, and that effective triplet interactions are consistently attractive. These results also imply that in the limit of zero macroion concentration $\left(n_{c}=n_{+}-n_{-} \rightarrow 0\right)$, or of high salt concentration $\left(n_{\mu} \rightarrow \infty\right)$, such that $\left(n_{+}-n_{-}\right) / n_{\mu} \rightarrow 0$, the leading-order nonlinear corrections all vanish. This dilute limit follows naturally from the fact that, for a pure symmetric electrolyte, response functions related by symmetry are equal $\left(\chi_{+++}^{\prime}=\chi_{---}^{\prime}, \chi_{++-}^{\prime}=\chi_{+--}^{\prime}\right)$, and so, from Eq. (78), the nonlinear response function $\chi^{\prime}$ is zero. This result - a consequence of charge neutrality that is analogous to the vanishing of the first nonlinear (quadratic) term in the expansion of the nonlinear PB equation - may partially explain the surprisingly broad range of validity of DLVO theory for high-ionic-strength suspensions. Nevertheless, even in dilute suspensions at high ionic strength, higher-order nonlinear corrections do not necessarily vanish. For this reason, predictions of the first-order nonlinear theory in the dilute limit may deviate from numerical solutions of the full nonlinear PB equation, which include, by construction, nonlinear corrections to all orders. The first-order corrections are nonetheless valuable in signaling the onset of nonlinearity, as shown below.

Equations (81) and (82) may be compared with related expressions derived via the density-functional approach. Equation (81) is similar in structure to an expression for a wall-induced effective pair interaction derived by Goulding and Hansen [56, 57] (Eq. (13) of Ref. [56]) if one factor of $\rho_{1}(r)$ in Eq. [81] is replaced by a wall counterion density orbital. It should be noted that these authors neglected the bulk nonlinear correction derived here [Eq. (81)], which is justified only in the dilute limit of an isolated pair of macroions. Equation (82) is similar to an expression for the triplet interaction derived by Löwen and Allahyarov [58], differing only by a factor of $\left(n_{+}-n_{-}\right) / n_{\mu}$ and by our excluded-volume correction in the definition of $\kappa$.

\section{E. Analytical Expressions for Effective Interactions}

Quantitative predictions of nonlinear response theory are facilitated by reducing the effective interactions to computationally practical analytical forms. Substituting Eq. (65) into Eq. (80), and evaluating the integrals, the nonlinear correction to the volume energy can be expressed as

$$
\Delta E=\frac{N_{m} k_{B} T}{6} \frac{\left(n_{+}-n_{-}\right)}{n_{\mu}^{3}}\left[\frac{Z^{2} \kappa^{3} n_{\mu}}{8 \pi}\left(\frac{1}{1+\kappa a}\right)^{2}-\frac{Z^{3} \kappa^{6}}{(4 \pi)^{2}}\left(\frac{e^{\kappa a}}{1+\kappa a}\right)^{3} \mathrm{E}_{1}(3 \kappa a)\right]
$$


where $\mathrm{E}_{1}$ is the exponential integral function [71]

$$
\mathrm{E}_{1}(x)=\int_{1}^{\infty} \mathrm{d} u \frac{e^{-x u}}{u}, \quad x>0 .
$$

Similarly, the nonlinear correction to the effective pair potential can be rendered analytically. The key is expressing the integral $I_{1}(r) \equiv \int \mathrm{d} \mathbf{r}^{\prime} \rho_{1}\left(r^{\prime}\right)\left[\rho_{1}\left(\left|\mathbf{r}-\mathbf{r}^{\prime}\right|\right)\right]^{2}$ in Eq. (81) in the form $I_{1}=\mathcal{F}^{-1}\left\{\hat{\rho}_{1}(k) \hat{\rho}_{2}(k)\right\}$, where $\hat{\rho}_{2}(k) \equiv \mathcal{F}\left\{\left[\rho_{1}(r)\right]^{2}\right\}$, with $\mathcal{F}$ denoting the Fourier transform operator and $\mathcal{F}^{-1}$ its inverse. Substituting Eq. (65) into Eq. (81), $\Delta v_{\text {eff }}^{(2)}(r)$ reduces, after some algebra, to

$$
\Delta v_{\mathrm{eff}}^{(2)}(r)=f_{1}(r) \frac{e^{-\kappa r}}{r}+f_{2}(r) \frac{e^{\kappa r}}{r}+f_{3}(r) \frac{e^{-\kappa a}}{r}, \quad r>\sigma,
$$

where

$$
\begin{gathered}
f_{1}(r)=C_{1}\left[\kappa(r-\sigma)+1-e^{-\kappa \sigma}\right]+C_{2}\left[\mathrm{E}_{1}(\kappa(r-a))+\mathrm{E}_{1}(3 \kappa a)-\mathrm{E}_{1}(\kappa a)\right], \\
f_{2}(r)=-C_{2} \mathrm{E}_{1}(3 \kappa(r+a)),
\end{gathered}
$$

and

$$
f_{3}(r)=C_{2}\left[\mathrm{E}_{1}(2 \kappa(r+a))-\mathrm{E}_{1}(2 \kappa(r-a))\right]
$$

with

$$
C_{1}=\frac{1}{6} \frac{\left(n_{+}-n_{-}\right)}{n_{\mu}} \frac{Z^{2} e^{2}}{\epsilon}\left(\frac{e^{\kappa a}}{1+\kappa a}\right)^{2}
$$

and

$$
C_{2}=\frac{1}{8 \pi} \frac{\left(n_{+}-n_{-}\right)}{n_{\mu}^{2}} \frac{Z^{3} e^{2} \kappa^{3}}{z \epsilon}\left(\frac{e^{\kappa a}}{1+\kappa a}\right)^{3} .
$$

It is interesting to examine the asymptotic $(r \rightarrow \infty)$ behavior of the leading-order nonlinear response approximation to the effective pair interaction. From Eqs. (85)-(88), using the inequality $\mathrm{E}_{1}(x)<e^{-x} / x$, we find the asymptotic limit

$$
\lim _{r \rightarrow \infty} v_{\mathrm{eff}}^{(2)}(r)=C_{1} \kappa e^{-\kappa r}
$$

which exhibits a more gradual decay than the screened-Coulomb DLVO potential [Eq. [67)]. We emphasize that this result does not contradict measurements of DLVO-like interactions between isolated pairs of macroions in dilute suspensions [12], since in the dilute limit $C_{1} \rightarrow 0$ and the asymptotic behavior reduces to that of linear response. The physical significance of Eq. (91) may be limited, however, by the neglect of higher-order nonlinear terms and shielding by intervening macroions 72 at distances beyond the mean nearest-neighbor separation, $r>\left[3 /\left(4 \pi n_{m}\right)\right]^{1 / 3}$.

\section{NUMERICAL INVESTIGATIONS AND DISCUSSION}

To quantitatively illustrate the influence of nonlinear screening, we compute counterion density profiles and effective pair and triplet interactions for selected system parameters. All results presented are for the case of monovalent counterions $(z=1)$ and aqueous suspensions at room temperature $\left(\lambda_{B}=0.714 \mathrm{~nm}\right)$. Figure 3 compares the counterion density profile around a single macroion of diameter $\sigma=100 \mathrm{~nm}$ and valences $Z=100$ and 500 in a dilute $(\eta=0.01)$ salt-free suspension, as predicted by linear response (DLVO) theory [Eq. (58)] and by first-order nonlinear response theory [Eq. (60)]. The linear-response counterion density $\rho_{c 0}(\mathbf{r})$ is approximated here by a single orbital $\rho_{1}(r)$ [Eq. (65)]. Evidently, nonlinear response sharpens the distribution of counterions around a macroion. 
Figure 4 compares the linear and nonlinear response predictions for the effective pair interaction, now for $Z=400$ (a) and 700 (b) and with small concentrations of added salt. As a check on the calculations, the interactions were computed both by Monte Carlo integration of Eq. (81) and from the analytical expressions [Eqs. [85)-(90)], the results being identical to within numerical error. Enhanced screening, resulting from the sharper nonlinear counterion profiles around macroions, has the general effect of weakening the pair interactions. For a given macroion diameter, nonlinear corrections increase in magnitude with increasing macroion valence and concentration and with decreasing salt concentration. Qualitatively, our predictions are consistent with the recent observations of Durand and Franck [73] of surprisingly short-ranged pair correlations in highly deionized colloidal suspensions. A quantitative comparison, however, would require computing the radial distribution function $g(r)$ from our $v_{\text {eff }}^{(2)}(r)$, by means of integral-equation theory or simulation, or computing $v_{\text {eff }}^{(2)}(r)$ from the experimental $g(r)$ data. Note that the effective pair potential discussed here is distinct from the potential of mean force $v_{\mathrm{mf}}(r)$, which was obtained in ref. 73] from the experimentally measured $g(r)$ via the definition $v_{\mathrm{mf}}(r)=-k_{B} T \ln g(r)$.

In Fig. 4t, the parameters (macroion diameter, $\sigma=652 \mathrm{~nm}$, and volume fraction, $\eta=0.0352$ ) are chosen to compare with the experiments of Larsen and Grier 10] in which unusually long-lived metastable fcc crystallites were observed. The macroion valence here is set to the maximum consistent with charge renormalization 74 ], $Z^{*} \sim O(10)\left(a / \lambda_{B}\right) \simeq 5000$, assuming the charge of a macroion to be reduced in bulk compared with its value in isolation 75 . While the pair interaction remains repulsive, it is significantly weaker than the DLVO prediction over a range comparable to the macroion diameter. A weaker pair interaction could promote the influence of three-body attractions, as well as interactions ignored by meanfield theory, such as short-range counterion fluctuation-induced attractions [25, 40]. In this way, nonlinear response may contribute to explaining experimental evidence for apparent attractions between like-charged macroions. The phase behavior of highly-charged colloidal crystals will be the subject of a future study.

The insets to Fig. 4 illustrate the extent to which the effective pair interaction may be fit by a screenedCoulomb (DLVO) potential. For sufficiently weak nonlinearity (Fig. 4a), $v_{\text {eff }}^{(2)}(r)$ may be reasonably fit by a DLVO potential with the same screening constant but a lower (renormalized) effective charge. The tendency of nonlinear screening to preserve the DLVO form of potential is consistent with conclusions from PB cell model calculations [4], ab initio simulations [21, 22], and optical tweezer experiments [10], all of which support the bulk DLVO potential in the weakly nonlinear regime. In the more strongly nonlinear regime (Figs. 4b and 4r), however, our calculations indicate that effective pair interactions may deviate significantly from DLVO form. In this regime, if $v_{\text {eff }}^{(2)}(r)$ can be fit at all by a DLVO potential, then it is only over a limited range and only by allowing renormalization of both the charge and screening constant. Similar departures from DLVO behavior with increasing macroion-counterion coupling strength have been predicted by integral-equation theories [43, 44, 45, 46, 47, 48, 49].

Although in the physically relevant range of macroion valence $\left(Z<Z^{*}\right)$ the predicted pair interaction is always purely repulsive, at higher (unphysical) valences $\left(Z>Z^{*}\right)$ the leading-order nonlinear theory predicts that $v_{\mathrm{eff}}^{(2)}(r)$ can develop an attractive well at sufficiently high macroion concentrations. Mathematical proofs [76, 77, 78] have recently shown, however, that PB theory cannot yield pair attraction - at least between a pair of isolated macroions. Since the RPA used here is formally equivalent to mean-field PB theory (see Appendix), the emergence of an attractive pair potential is best interpreted as a sign that higher-order nonlinear terms must then be included.

As a quantitative test of the nonlinear response theory, we compare predictions with available data from the ab initio simulations of Tehver et al. 21]. By assuming a counterion density orbital and ignoring counterion density fluctuations, the $a b$ initio approach provides the most direct test of the theory. Figure 5 a presents the comparison for the total potential energy of interaction between a pair of macroions, of diameter $\sigma=106 \mathrm{~nm}$ and valence $Z=200$, in a cubic box of length $530 \mathrm{~nm}$ with periodic boundary conditions (taking into account image interactions) in the absence of salt. The theory is in essentially perfect agreement with simulation, although nonlinear effects, for these parameters, are relatively weak. Figure [5] shows results for a higher valence $(Z=700)$, for which case simulation data are not yet available, but where nonlinear effects are more prominent. Further simulations of more highly charged macroions would more severely test the theory - in particular, convergence of the perturbation expansion - in the strongly nonlinear regime.

To quantify the range of validity of the linear response approximation and to measure the impact of nonlinear screening on thermodynamics, we calculate the magnitude of the leading-order nonlinear correction to the pair interaction $\Delta v_{\text {eff }}^{(2)}(r)$ at the mean nearest-neighbor separation $r_{\mathrm{nn}}$, where pair interactions contribute 
most to the potential energy. Figure 6 maps out, in the space of macroion volume fraction $\eta$ and salt concentration $c_{s}$ (measured in $\mu \mathrm{M}$ ), the boundary of the region within which $\left|\Delta v_{\text {eff }}^{(2)}\left(r_{\mathrm{nn}}\right)\right|$ exceeds typical thermal energies, for the fcc crystal structure: $r_{\mathrm{nn}} / \sigma=2^{-1 / 2}(2 \pi / 3 \eta)^{1 / 3}$. For points above the boundary curves, $\left|\Delta v_{\text {eff }}^{(2)}\left(r_{\mathrm{nn}}\right)\right|>1 k_{B} T$ (Fig. [6 a ) or $0.1 k_{B} T$ (Fig. 6b). Points on the boundary curves in Fig. [6 correspond to thermodynamic states for which a stable fcc crystal phase is predicted by simulations of model Yukawa systems [17], although these simulations do not include influences of the volume energy. With increasing $Z$ and decreasing $c_{s}$, the threshold $\eta$ decreases. Thus, nonlinear screening is anticipated to increasingly influence thermodynamics with increasing macroion charge and concentration and with decreasing ionic strength - just the conditions under which anomalous phase behavior has been observed [, 6 , [8, 9, 10].

Moving beyond pair interactions, Fig. 7 shows the effective three-body interaction between a triplet of macroions arranged in an equilateral triangle for $\sigma=100 \mathrm{~nm}$ and two different valences, $Z=500$ and 700. The interactions were computed numerically by Monte Carlo integration of Eq. 82]. The strength of the interaction is seen to grow rapidly with increasing macroion valence and with decreasing separation between macroion cores. In a concentrated suspension of highly-charged macroions, effective many-body interactions may become significant. In particular, as noted above, triplet attractions may well contribute to the surprising metastability of colloidal crystallites observed in deionized suspensions [10].

To again test the theory against simulation, we compute the force on a macroion in an equilateral-triangle configuration of three macroions as a function of the triangle edge length. On expanding a triangle from edge length $R-\Delta R / 2$ to $R+\Delta R / 2$, the energy changes by

$$
\Delta U=3\left[v_{\mathrm{eff}}^{(2)}\left(R+\frac{\Delta R}{2}\right)-v_{\mathrm{eff}}^{(2)}\left(R-\frac{\Delta R}{2}\right)\right]+v_{\mathrm{eff}}^{(3)}\left(R+\frac{\Delta R}{2}\right)-v_{\mathrm{eff}}^{(3)}\left(R-\frac{\Delta R}{2}\right) .
$$

Since, as the triangle expands, each of the three macroions moves a distance $\Delta R / \sqrt{3}$ parallel to the total effective force $F$ acting on it, the change in energy also may be expressed as $\Delta U=-3 F \Delta R / \sqrt{3}$. Equating the two expressions for $\Delta U$, the total force can be written as

$$
F(R)=F^{(2)}(R)+F^{(3)}(R),
$$

where

$$
F^{(2)}(R)=\frac{-\sqrt{3}}{\Delta R}\left[v_{\mathrm{eff}}^{(2)}\left(R+\frac{\Delta R}{2}\right)-v_{\mathrm{eff}}^{(2)}\left(R-\frac{\Delta R}{2}\right)\right]
$$

and

$$
F^{(3)}(R)=\frac{-1}{\sqrt{3} \Delta R}\left[v_{\text {eff }}^{(3)}\left(R+\frac{\Delta R}{2}\right)-v_{\text {eff }}^{(3)}\left(R-\frac{\Delta R}{2}\right)\right]
$$

are the effective pair and triplet forces, respectively.

To compare directly with available simulation data 21], we consider macroions of diameter $\sigma=106 \mathrm{~nm}$ in a cubic box of length $1000 \mathrm{~nm}$ with periodic boundary conditions in the absence of salt. Over a range of macroion valences, we compute the sum of linear (DLVO) effective pair forces, the sum of nonlinear effective pair forces, the effective triplet force, and the total effective force (sum of pair and triplet forces), from Eqs. (931)-(95). For a valence of $Z=200$ - the only case for which simulation data were reported [21] the predicted total force is essentially identical to the sum of pair forces, consistent with ref. 21], in which an absence of many-body effects was concluded. For higher valences, however, three-body forces are not negligible. Figure 8 presents predictions of the theory for $Z=700$ and 1000 , demonstrating significant deviations of the total force from the sum of pair forces. For the case $Z=1000$, which somewhat exceeds the charge-renormalization limit [4], the predicted total force actually becomes attractive beyond $r \simeq 2 \sigma$, although this is likely an artifact of truncating the perturbation series and thereby neglecting higher-order nonlinear terms. Again, further simulations could help to resolve the issue.

Other workers have investigated many-body interactions in charged colloids. Schmitz 79 has developed a theory that describes sharing of counterions between macroions, analogous to molecular chemical bonding, and used the theory to study the influence of many-body effects on counterion distributions and the structure of colloidal crystals. Sear [80], exploring a phenomenological model, showed that interactions among particles 
with internal degrees of freedom are, in general, non-pairwise-additive, and that triplet interactions may be attractive at the same time that pair interactions are repulsive. Recently, Russ et al. [81] solved the nonlinear $\mathrm{PB}$ equation for triplets of macroions immersed in an electrolyte. Their conclusion that three-body effects are always cohesive agrees qualitatively with our results, and those of ref. [58]. We note, however, that three-body contributions to the grand potential, calculated in ref. [81], are not directly comparable to threebody interactions in the effective Hamiltonian, calculated here and in the simulations of Tehver et al. 21]. In another study, Wu et al. [82] extracted three-body forces from Monte Carlo simulations of macroion triplets in equilateral configurations. These authors found attractive electrostatic three-body forces, but also detected a significant repulsive contribution attributable to hard-sphere collisions between macroions and microions, which were modelled in the simulations as charged hard spheres. Future extension of the response theory from point microions to hard-core microions would allow a more direct comparison with these Monte Carlo data.

Influences on thermodynamic phase behavior of nonlinear corrections to both effective interactions and the volume energy are now being explored. It may be anticipated that these corrections will be especially significant for deionized suspensions of highly charged macroions. Preliminary calculations of free energies and phase diagrams [83] indicate that the spinodal-instability mechanism proposed to describe phase separation [30, 31] remains qualitatively valid - at least under the assumption of fixed macroion charge - but that nonlinearity can significantly shift the phase boundaries and, in some cases, enhance the tendency toward phase separation.

The role of nonlinear response and of effective many-body interactions in dense electron-ion (metallic) systems has long been recognized and discussed [84, 85, 86, 87, 88]. In this context, it has been argued that nonlinear corrections to pair potentials and structure factors either are weak 88 or can be incorporated into the linear response scheme [87], but that nonlinear corrections to the volume energy and thermodynamic properties (e.g., bulk modulus) are more significant [86]. Whether the same argument applies also to colloidal systems is being investigated in ongoing studies of phase behavior [83].

\section{SUMMARY AND CONCLUSIONS}

In summary, by incorporating nonlinear microion screening into a mean-field response theory of charged colloids in the primitive model, we have derived nonlinear corrections to the effective electrostatic interactions between hard spherical macroions in bulk colloidal suspensions. The key physical concept is that nonlinear screening entails both effective many-body interactions and essential corrections to the effective pair potential and the one-body volume energy. Effective triplet interactions are predicted to be always attractive, consistent with previous work [58, 81]. The effective pair potential $v_{\text {eff }}^{(2)}(r)$, which in the linear (DLVO) theory has screened-Coulomb form, is shortened in range by the influence of nonlinear screening, but remains purely repulsive within the physically reasonable range of renormalized macroion charges. Predictions for $v_{\text {eff }}^{(2)}(r)$ are in essentially perfect agreement with available $a b$ initio simulation data [21]. The theory also predicts that triplet forces are negligible between weakly charged macroions, consistent with simulation [21], but can be significant for higher macroion charges. Further simulations of more highly charged and concentrated macroions are now required to more severely test the theory.

Analytical and numerical results confirm that nonlinear effects become qualitatively stronger with increasing macroion charge, increasing macroion concentration, and decreasing salt concentration. In the dilute limit of zero macroion concentration, but nonzero salt concentration, leading-order nonlinear corrections vanish. Perhaps the most practical application of the response theory, illustrated here, is to mapping out the parameter ranges within which linear theories can be trusted. Future work will explore implications of nonlinear screening for thermodynamic properties, in particular, the phenomenon of phase separation at low salt concentrations and the stability of deionized charged colloidal crystals.

\section{Acknowledgments}

Helpful discussions and correspondence with Juan Antonio Anta, Neil Ashcroft, Jayanth Banavar, Carl Franck, Christos Likos, Hartmut Löwen, Kenneth Schmitz, and Hao Wang are gratefully acknowledged. This work was supported by the National Science Foundation under Grant Nos. DMR-0204020 and EPS-0132289. 


\section{APPENDIX: COMPARISON WITH RELATED THEORETICAL APPROACHES}

\section{Response Theory vs. Poisson-Boltzmann Theory}

We demonstrate here that response theory, when combined with the random phase approximation (RPA) for the microion response functions (see Sec. IIIE) is formally equivalent to Poisson-Boltzmann theory. The ensemble-averaged number density profile of positive microions, in the presence of the macroion potential $\phi_{\text {ext }}(\mathbf{r})$, is given exactly by the Euler-Lagrange equation [63, 89],

$$
\rho_{+}(\mathbf{r})=\frac{e^{\beta \mu_{+}}}{\Lambda_{+}^{3}} \exp \left[-\beta z e \phi_{\operatorname{ext}}(\mathbf{r})+c_{+}^{(1)}\left(\mathbf{r} ;\left[\rho_{+}(\mathbf{r}), \rho_{-}(\mathbf{r})\right]\right)\right],
$$

which follows from minimization of the grand potential functional with respect to $\rho_{+}(\mathbf{r})$. In Eq. A.1), $\mu_{+}$ and $\Lambda_{+}$denote the chemical potential and thermal de Broglie wavelength of the positive microions, $\phi_{\text {ext }}(\mathbf{r})$ is the "external" electrostatic potential of the macroions, and $c_{+}^{(1)}\left(\mathbf{r} ;\left[\rho_{+}(\mathbf{r}), \rho_{-}(\mathbf{r})\right]\right)$ is the one-particle direct correlation function (DCF) of the positive microions, which is a functional of the inhomogeneous microion densities. Expanding $c_{+}^{(1)}\left(\mathbf{r} ;\left[\rho_{+}(\mathbf{r}), \rho_{-}(\mathbf{r})\right]\right)$ in a functional Taylor series about the average (bulk) microion densities, $n_{+}$and $n_{-}$, we have

$$
\begin{aligned}
\rho_{+}(\mathbf{r}) & =\frac{e^{\beta \mu_{+}}}{\Lambda_{+}^{3}} \exp \left[-\beta z e \phi_{\operatorname{ext}}(\mathbf{r})+c_{+}^{(1)}\left(n_{+}, n_{-}\right)+\int \mathrm{d} \mathbf{r}^{\prime} c_{++}^{(2)}\left(\left|\mathbf{r}-\mathbf{r}^{\prime}\right| ; n_{+}, n_{-}\right)\left[\rho_{+}\left(\mathbf{r}^{\prime}\right)-n_{+}\right]\right. \\
& \left.+\int \mathrm{d} \mathbf{r}^{\prime} c_{+-}^{(2)}\left(\left|\mathbf{r}-\mathbf{r}^{\prime}\right| ; n_{+}, n_{-}\right)\left[\rho_{-}\left(\mathbf{r}^{\prime}\right)-n_{-}\right]+\cdots\right]
\end{aligned}
$$

where $c_{i j}^{(2)}\left(r ; n_{+}, n_{-}\right), i, j= \pm$, are the bulk microion two-particle DCFs, which are related to the one-particle DCFs via

$$
c_{i j}^{(2)}\left(\left|\mathbf{r}-\mathbf{r}^{\prime}\right| ; n_{+}, n_{-}\right)=\lim _{\rho_{ \pm}(\mathbf{r}) \rightarrow n_{ \pm}}\left[\frac{\delta c_{i}^{(1)}\left(\mathbf{r} ;\left[\rho_{+}(\mathbf{r}), \rho_{-}(\mathbf{r})\right]\right)}{\delta \rho_{j}\left(\mathbf{r}^{\prime}\right)}\right] .
$$

We now make the mean-field random phase approximation 63]: (1) neglecting three-particle and higherorder correlations, i.e., truncating the series in Eq. (A.2), and (2) ignoring short-range pair correlations by simply equating $c_{i j}^{(2)}\left(r ; n_{+}, n_{-}\right)$to their asymptotic long-range limits,

$$
c_{i j}^{(2)}\left(r ; n_{+}, n_{-}\right) \simeq-\beta v_{i j}(r), \quad i, j= \pm .
$$

Equation A.2 then becomes

$$
\begin{aligned}
& \rho_{+}(\mathbf{r}) \simeq n_{+} \exp {\left[-\beta\left(z e \phi_{\operatorname{ext}}(\mathbf{r})+\int \mathrm{d} \mathbf{r}^{\prime} v_{++}\left(\left|\mathbf{r}-\mathbf{r}^{\prime}\right|\right)\left[\rho_{+}\left(\mathbf{r}^{\prime}\right)-n_{+}\right]\right.\right.} \\
&\left.\left.+\int \mathrm{d} \mathbf{r}^{\prime} v_{+-}\left(\left|\mathbf{r}-\mathbf{r}^{\prime}\right|\right)\left[\rho_{-}\left(\mathbf{r}^{\prime}\right)-n_{-}\right]\right)\right] \\
&=n_{+} \exp [-\beta z e \phi(\mathbf{r})],
\end{aligned}
$$

where we have used $n_{+}=\left(e^{\beta \mu_{+}} / \Lambda_{+}^{3}\right) \exp \left[c_{+}^{(1)}\left(n_{+}, n_{-}\right)\right]$and have identified

$$
\phi(\mathbf{r})=\phi_{\text {ext }}(\mathbf{r})+\int \mathrm{d} \mathbf{r}^{\prime} \frac{z e}{\epsilon\left|\mathbf{r}-\mathbf{r}^{\prime}\right|}\left[\rho_{+}\left(\mathbf{r}^{\prime}\right)-n_{+}-\rho_{-}\left(\mathbf{r}^{\prime}\right)+n_{-}\right]
$$

as the total electrostatic potential, due to both the macroions and the surrounding microions. Similarly, the density profile of negative microions is given by

$$
\begin{aligned}
\rho_{-}(\mathbf{r}) \simeq n_{-} \exp & {\left[-\beta\left(-z e \phi_{\mathrm{ext}}(\mathbf{r})+\int \mathrm{d} \mathbf{r}^{\prime} v_{+-}\left(\left|\mathbf{r}-\mathbf{r}^{\prime}\right|\right)\left[\rho_{+}\left(\mathbf{r}^{\prime}\right)-n_{+}\right]\right.\right.} \\
& \left.\left.+\int \mathrm{d} \mathbf{r}^{\prime} v_{--}\left(\left|\mathbf{r}-\mathbf{r}^{\prime}\right|\right)\left[\rho_{-}\left(\mathbf{r}^{\prime}\right)-n_{-}\right]\right)\right] \\
= & n_{-} \exp [\beta z e \phi(\mathbf{r})] .
\end{aligned}
$$


Combining Eqs. (A.5) and (A.7) with the Poisson equation

$$
\nabla^{2} \phi(\mathbf{r})=-\frac{4 \pi}{\epsilon}\left\{z e \rho_{+}(\mathbf{r})-z e \rho_{-}(\mathbf{r})\right\}
$$

we obtain

$$
\nabla^{2} \phi(\mathbf{r})=-\frac{4 \pi z e}{\epsilon}\left\{n_{+} \exp [-\beta z e \phi(\mathbf{r})]-n_{-} \exp [\beta z e \phi(\mathbf{r})]\right\},
$$

which is the $\mathrm{PB}$ equation for macroions in a symmetric $z: z$ electrolyte. We conclude that the RPA-based response theory is formally equivalent to the mean-field PB theory. This is not surprising, given that both approaches neglect microion correlations. Response theory, however, provides a powerful framework for going beyond a mean-field description by systematically including microion correlations via more accurate approximations for the response functions of the microion plasma.

\section{Response Theory vs. Integral Equation Theory}

Here we show that linear response theory is equivalent to a linearized hypernetted-chain (HNC) approximation in integral-equation theory. Substituting Eqs. (72.74) into the Fourier transform of Eq. (75), the linear response expressions for the ensemble-averaged number density profiles of positive and negative microions are

$$
\frac{\rho_{+}(\mathbf{r})}{n_{+}}=1-\beta \sum_{i}\left[v_{m+}\left(\left|\mathbf{r}-\mathbf{R}_{i}\right|\right)+\int \mathrm{d} \mathbf{r}^{\prime}\left[n_{+} h_{++}\left(\left|\mathbf{r}-\mathbf{r}^{\prime}\right|\right)-n_{-} h_{+-}\left(\left|\mathbf{r}-\mathbf{r}^{\prime}\right|\right)\right] v_{m+}\left(\left|\mathbf{r}^{\prime}-\mathbf{R}_{i}\right|\right)\right]
$$

and

$$
\frac{\rho_{-}(\mathbf{r})}{n_{-}}=1+\beta \sum_{i}\left[v_{m+}\left(\left|\mathbf{r}-\mathbf{R}_{i}\right|\right)+\int \mathrm{d} \mathbf{r}^{\prime}\left[n_{-} h_{--}\left(\left|\mathbf{r}-\mathbf{r}^{\prime}\right|\right)-n_{+} h_{+-}\left(\left|\mathbf{r}-\mathbf{r}^{\prime}\right|\right)\right] v_{m+}\left(\left|\mathbf{r}^{\prime}-\mathbf{R}_{i}\right|\right)\right] .
$$

On the other hand, from Eq. A.2, we have the exact relations

$$
\begin{aligned}
\rho_{+}(\mathbf{r}) & =n_{+} \exp \left[-\beta \sum_{i} v_{m+}\left(\left|\mathbf{r}-\mathbf{R}_{i}\right|\right)+\int \mathrm{d} \mathbf{r}^{\prime} c_{++}^{(2)}\left(\left|\mathbf{r}-\mathbf{r}^{\prime}\right|\right)\left[\rho_{+}\left(\mathbf{r}^{\prime}\right)-n_{+}\right]\right. \\
& \left.+\int \mathrm{d} \mathbf{r}^{\prime} c_{+-}^{(2)}\left(\left|\mathbf{r}-\mathbf{r}^{\prime}\right|\right)\left[\rho_{-}\left(\mathbf{r}^{\prime}\right)-n_{-}\right]+\cdots\right]
\end{aligned}
$$

and

$$
\begin{aligned}
\rho_{-}(\mathbf{r}) & =n_{-} \exp \left[\beta \sum_{i} v_{m+}\left(\left|\mathbf{r}-\mathbf{R}_{i}\right|\right)+\int \mathrm{d} \mathbf{r}^{\prime} c_{--}^{(2)}\left(\left|\mathbf{r}-\mathbf{r}^{\prime}\right|\right)\left[\rho_{-}\left(\mathbf{r}^{\prime}\right)-n_{-}\right]\right. \\
& \left.+\int \mathrm{d} \mathbf{r}^{\prime} c_{+-}^{(2)}\left(\left|\mathbf{r}-\mathbf{r}^{\prime}\right|\right)\left[\rho_{+}\left(\mathbf{r}^{\prime}\right)-n_{+}\right]+\cdots\right] .
\end{aligned}
$$

Truncating the expansions on the right side of Eqs. A.12 and A.13) at the level of two-particle correlations amounts to the HNC approximation in integral-equation theory. If we further linearize the exponential functions (expanding and neglecting all but the first two terms), substitute recursively for $\rho_{+}(\mathbf{r})$ and $\rho_{-}(\mathbf{r})$, and use the Ornstein-Zernike (OZ) relation for mixtures [63]

$$
h_{i j}(r)=c_{i j}^{(2)}(r)+\sum_{k} n_{k} \int \mathrm{d} \mathbf{r}^{\prime} c_{i k}^{(2)}\left(\left|\mathbf{r}-\mathbf{r}^{\prime}\right|\right) h_{k j}\left(r^{\prime}\right),
$$

we recover Eqs. A.10 and A.11). Thus, the linear response approximation is equivalent to a linearized-HNC closure of the $\mathrm{OZ}$ relation, while nonlinear response generates new closures. 
[1] R. J. Hunter, Foundations of Colloid Science (Oxford University Press, Oxford, 1986).

[2] P. N. Pusey, in Liquids, Freezing and Glass Transition, session 51, ed. J.-P. Hansen, D. Levesque, and J. ZinnJustin (North-Holland, Amsterdam, 1991).

[3] K. S. Schmitz, Macroions in Solution and Colloidal Suspension (VCH, New York, 1993).

[4] F. Oosawa, Polyelectrolytes (Dekker, New York, 1971).

[5] Polyelectrolytes, ed. M. Hara (Dekker, New York, 1993).

[6] B. V. R. Tata, M. Rajalakshmi, and A. K. Arora, Phys. Rev. Lett. 69, 3778 (1992).

[7] K. Ito, H. Yoshida, and N. Ise, Science 263, 66 (1994).

[8] H. Matsuoka, T. Harada, and H. Yamaoka, Langmuir 10, 4423(1994); H. Matsuoka, T. Harada, K. Kago, and H. Yamaoka, ibid 12, 5588 (1996); T. Harada, H. Matsuoka, T. Ikeda, and H. Yamaoka, ibid 15, 573 (1999).

[9] F. Gröhn and M. Antonietti, Macromolecules 33, 5938 (2000).

[10] A. E. Larsen and D. G. Grier, Nature 385, 230 (1997).

[11] G. M. Kepler and S. Fraden, Phys. Rev. Lett. 73, 356 (1994).

[12] A. E. Larsen and D. G. Grier, Phys. Rev. Lett. 76, 3862 (1996); J. C. Crocker and D. G. Grier, Phys. Rev. Lett. 77, 1897 (1996).

[13] Y. Levin, Rep. Prog. Phys. 65, 1577 (2002).

[14] C. N. Likos, Phys. Rep. 348, 267 (2001).

[15] L. Belloni, J. Phys.: Condens. Matter 12, R549 (2000).

[16] J.-P. Hansen and H. Löwen, Ann. Rev. Phys. Chem. 51, 209 (2000).

[17] M. O. Robbins, K. Kremer, and G. G. Grest, J. Chem. Phys. 88, 3286 (1988).

[18] E. J. Meijer and D. Frenkel, J. Chem. Phys. 94, 2269 (1991).

[19] S. Auer and D. Frenkel, J. Phys.: Condens. Matter 14, 7667 (2002).

[20] A.-P. Hynninen and M. Dijkstra, Phys. Rev. E 68, 21407 (2003).

[21] R. Tehver, F. Ancilotto, F. Toigo, J. Koplik, and J. R. Banavar, Phys. Rev. E 59, R1335 (1999).

[22] H. Löwen, P. A. Madden, and J.-P. Hansen, Phys. Rev. Lett. 68, 1081 (1992); H. Löwen, J.-P. Hansen, and P. A. Madden, J. Chem. Phys. 98, 3275 (1993); H. Löwen and G. Kramposthuber, Europhys. Lett. 23, 673 (1993).

[23] M. J. Stevens, M. L. Falk, and M. O. Robbins, J. Chem. Phys. 104, 5209 (1996).

[24] P. Linse, J. Chem. Phys. 110, 3493 (1999); V. Lobaskin and P. Linse, J. Chem. Phys. 111, 4300 (1999); P. Linse and V. Lobaskin, Phys. Rev. Lett. 83, 4208 (1999); P. Linse, J. Chem. Phys. 113, 4359 (2000); J. Reščič and P. Linse, J. Chem. Phys. 114, 10131 (2001); V. Lobaskin, A. Lyubartsev, and P. Linse, Phys. Rev. E 63, 20401 (2001); V. Lobaskin and K. Qamhieh, J. Phys. Chem. B 107, 8022 (2003).

[25] R. Messina, C. Holm, and K. Kremer, Phys. Rev. Lett. 85, 872 (2000); Eur. Phys. J. E 4, 363 (2001).

[26] E. Allahyarov, I. D'Amico, and H. Löwen, Phys. Rev. Lett. 81, 1334 (1998).

[27] B. V. Derjaguin and L. Landau, Acta Physicochimica (USSR) 14, 633 (1941).

[28] E. J. W. Verwey and J. T. G. Overbeek, Theory of the Stability of Lyophobic Colloids (Elsevier, Amsterdam, 1948).

[29] H. Graf and H. Löwen, Phys. Rev. E 57, 5744 (1998).

[30] R. van Roij and J.-P. Hansen, Phys. Rev. Lett. 79, 3082 (1997).

[31] R. van Roij, M. Dijkstra, and J.-P. Hansen, Phys. Rev. E 59, 2010 (1999).

[32] M. J. Grimson and M. Silbert, Mol. Phys. 74, 397 (1991).

[33] A. R. Denton, J. Phys.: Condens. Matter 11, 10061 (1999).

[34] A. R. Denton, Phys. Rev. E 62, 3855 (2000).

[35] P. B. Warren, J. Chem. Phys. 112, 4683 (2000).

[36] D. Y. C. Chan, P. Linse, and S. N. Petris, Langmuir 17, 4202 (2001).

[37] I. Rouzina and V. A. Bloomfield, J. Phys. Chem. 100, 9977 (1996).

[38] B.-Y. Ha and A. J. Liu, Phys. Rev. Lett. 79, 1289 (1997); Phys. Rev. E 58, 6281 (1998).

[39] M. J. Stevens Phys. Rev. Lett. 82, 101 (1999); Biophys. J. 80, 130 (2001).

[40] B. I. Shklovskii, Phys. Rev. E 60, 5802 (1999); T. T. Nguyen, I. Rouzina, and B. I. Shklovskii, Phys. Rev. E 60, 7032 (1999).

[41] T. T. Nguyen, I. Rouzina, and B. I. Shklovskii, J. Chem. Phys. 112, 2562 (2000); T. T. Nguyen, A. Yu. Grosberg, and B. I. Shklovskii, J. Chem. Phys. 113, 1110 (2000).

[42] W. M. Gelbart, R. F. Bruinsma, P. A. Pincus, and V. A. Parsegian, Physics Today 53 (Sept. 2000), p. 38.

[43] G. N. Patey, J. Chem. Phys. 72, 5763 (1980).

[44] L. Belloni, Phys. Rev. Lett. 57, 2026 (1986).

[45] S. Khan and D. Ronis, Mol. Phys. 60, 637 (1987); S. Khan, T. L. Morton, and D. Ronis, Phys. Rev. A 35,4295 (1987).

[46] M. D. Carbajal-Tinoco and P. González-Mozuelos, J. Chem. Phys. 117, 2344 (2002). 
[47] L. B. Bhuiyan and C. W. Outhwaite, J. Chem. Phys. 116, 2650 (2002).

[48] S. N. Petris and D. Y. C. Chan, J. Chem. Phys. 116, 8588 (2002).

[49] J. A. Anta and S. Lago, J. Chem. Phys. 116, 10514 (2002); V. Morales, J. A. Anta, and S. Lago, Langmuir 19, 475 (2003).

[50] H. H. von Grünberg, R. van Roij, and G. Klein Europhys. Lett. 55, 580 (2001).

[51] M. Deserno and H. H. von Grünberg, Phys. Rev. E 66, 011401 (2002).

[52] M. N. Tamashiro and H. Schiessel, J. Chem. Phys. 119, 1855 (2003).

[53] W. R. Bowen and A. O. Sharif, Nature 393, 663 (1998).

[54] J. J. Gray, B. Chiang, and R. T. Bonnecaze, Nature 402, 750 (1999).

[55] J. Dobnikar, R. Rzehak, and H. H. von Grünberg, Europhys. Lett. 61, 695 (2003); J. Dobnikar, Y. Chen, R. Rzehak, and H. H. von Grünberg, J. Phys.: Condens. Matter 15, S263 (2003).

[56] D. Goulding and J.-P. Hansen, Europhys. Lett. 46, 407 (1999).

[57] J.-P. Hansen, D. Goulding, and R. van Roij, J. Phys. IV France 10, Pr5-27 (2000).

[58] H. Löwen and E. Allahyarov, J. Phys.: Condens. Matter 10, 4147 (1998).

[59] M. E. Fisher, J. Stat. Phys. 75, 1 (1994); X. Li, Y. Levin, and M. E. Fisher, Europhys. Lett. 26, 683 (1994); M. E. Fisher, Y. Levin, and X. Li, J. Chem. Phys. 101, 2273 (1994).

[60] N. V. Sushkin and G. D. J. Phillies, J. Chem. Phys. 103, 4600 (1995).

[61] L. E. González, D. J. González, M. Silbert, and S. Baer, Mol. Phys. 99, 875 (2001).

[62] J. S. Rowlinson, Mol. Phys. 52, 567 (1984).

[63] J.-P. Hansen and I. R. McDonald, Theory of Simple Liquids, $2^{\text {nd }}$ ed. (Academic, London, 1986).

[64] N. W. Ashcroft and D. Stroud, Solid State Phys. 33, 1 (1978).

[65] J. Hafner, From Hamiltonians to Phase Diagrams (Springer, Berlin, 1987).

[66] N. W. Ashcroft, Phys. Lett. 23, 48 (1966).

[67] We note that expansion about zero macroion charge $(u=0)$ in Eq. (16) is here the only natural choice, thus avoiding ambiguities encountered in other linearization schemes [50, 51, 52].

[68] E. A. Allahyarov, L. I. Podloubny, and P. P. J. M. Schram, and S. A. Trigger, Physica A 220, 349 (1995).

[69] We note that the counterion-counterion pair correlation function $h_{c c}(r)$ in Eq. (46) also appears in the distribution function approach of Chan et al. (cf. Eq. (45) of ref. [36]).

[70] Equation (79) has also been derived by a density-functional approach in refs. [29] and [31].

[71] I. S. Gradstein and I. M. Ryzhik, Table of Integrals, Series, and Products (Academic, New York, 1980).

[72] R. Klein, H. H. von Grünberg, C. Bechinger, M. Brunner, and V. Lobaskin, J. Phys.: Condens. Matter 14, 7631 (2002).

[73] R. V. Durand and C. Franck, Phys. Rev. E 61, 6922 (2000).

[74] S. Alexander, P. M. Chaikin, P. Grant, G. J. Morales, and P. Pincus, J. Chem. Phys. 80, 5776 (1984).

[75] In ref. [10], a macroion valence of $Z=7300$ is obtained by fitting the pair interaction between two isolated macroions with a DLVO potential. For bulk crystals, however, this valence somewhat exceeds the approximate charge-renormalization limit 74].

[76] J. C. Neu, Phys. Rev. Lett. 82, 1072 (1999).

[77] J. E. Sader and D. Y. C. Chan, J. Coll. Int. Sci. 213, 268 (1999); Langmuir 16, 324 (2000).

[78] E. Trizac and J. L. Raimbault, Phys. Rev. E 60, 6530 (1999); E. Trizac, Phys. Rev. E 62, R1465 (2000).

[79] K. S. Schmitz, Phys. Chem. Chem. Phys. 1, 2109 (1999).

[80] R. P. Sear, Phys. Rev. E 62, 2501 (2000).

[81] C. Russ, H. H. von Grünberg, M. Dijkstra, R. van Roij, Phys. Rev. E 66, 011402 (2002).

[82] J. Z. Wu, D. Bratko, H. W. Blanch, and J. M. Prausnitz, J. Chem. Phys. 113, 3360 (2000).

[83] A. R. Denton (unpublished).

[84] C. J. Pethick, Phys. Rev. B 2, 1789 (1970).

[85] E. G. Brovman and G. Solt, Solid State Comm. 8, 903 (1970).

[86] S. P. Singh and W. H. Young, J. Phys. F: Metal Phys. 3, 1127 (1973).

[87] M. Rasolt and R. Taylor, Phys. Rev. B 11, 2717 (1975); L. Dagens, M. Rasolt, and R. Taylor, Phys. Rev. B 11, 2726 (1975).

[88] A. A. Louis and N. W. Ashcroft, Phys. Rev. Lett. 81, 4456 (1998).

[89] From Eq. A.1 on, to simplify notation, we dispense with angular brackets in denoting ensemble-averaged densities. 


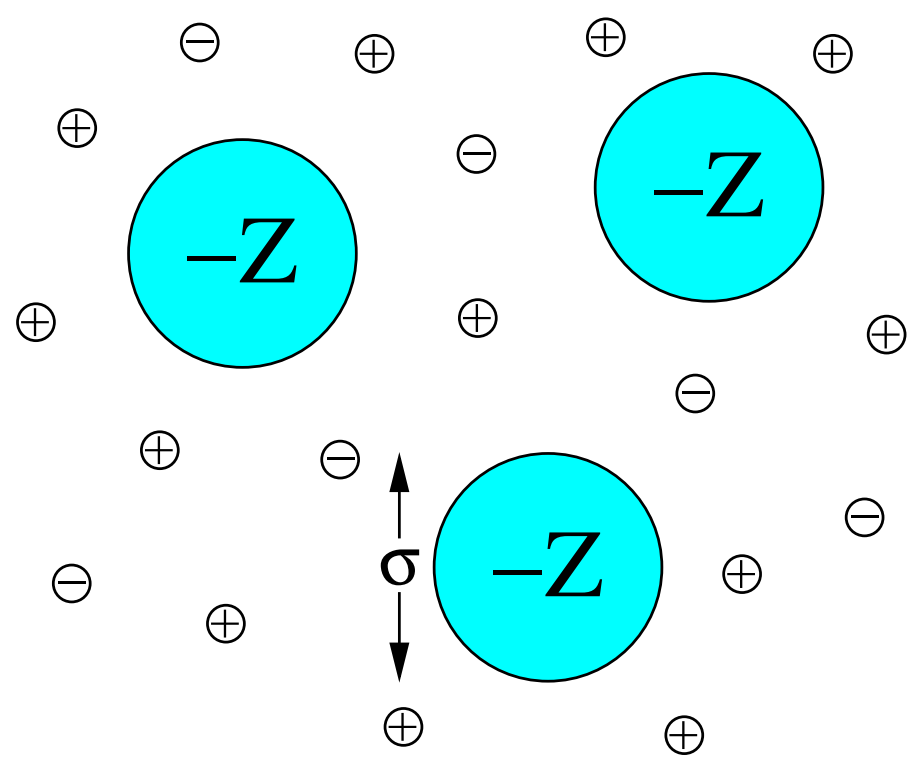

FIG. 1: Primitive model of a charged colloidal suspension: hard macroions (valence $-Z$ and diameter $\sigma$ ) and point microions (counterions and salt ions) in a dielectric continuum (not shown).

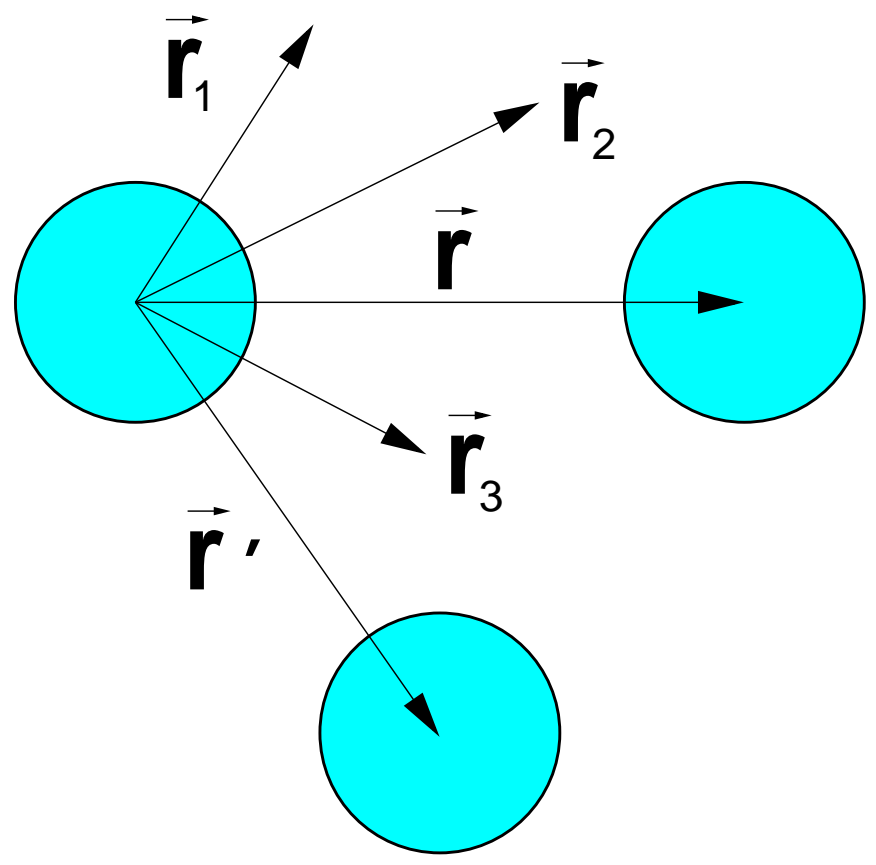

FIG. 2: Geometry for the physical interpretation of response theory (see Sec. IIID). Vectors $\mathbf{r}$ and $\mathbf{r}^{\prime}$ define centerto-center displacements of macroions. Vectors $\mathbf{r}_{1}, \mathbf{r}_{2}$, and $\mathbf{r}_{3}$ define points at which either the macroion "external" potential acts or a change in microion density is induced. 

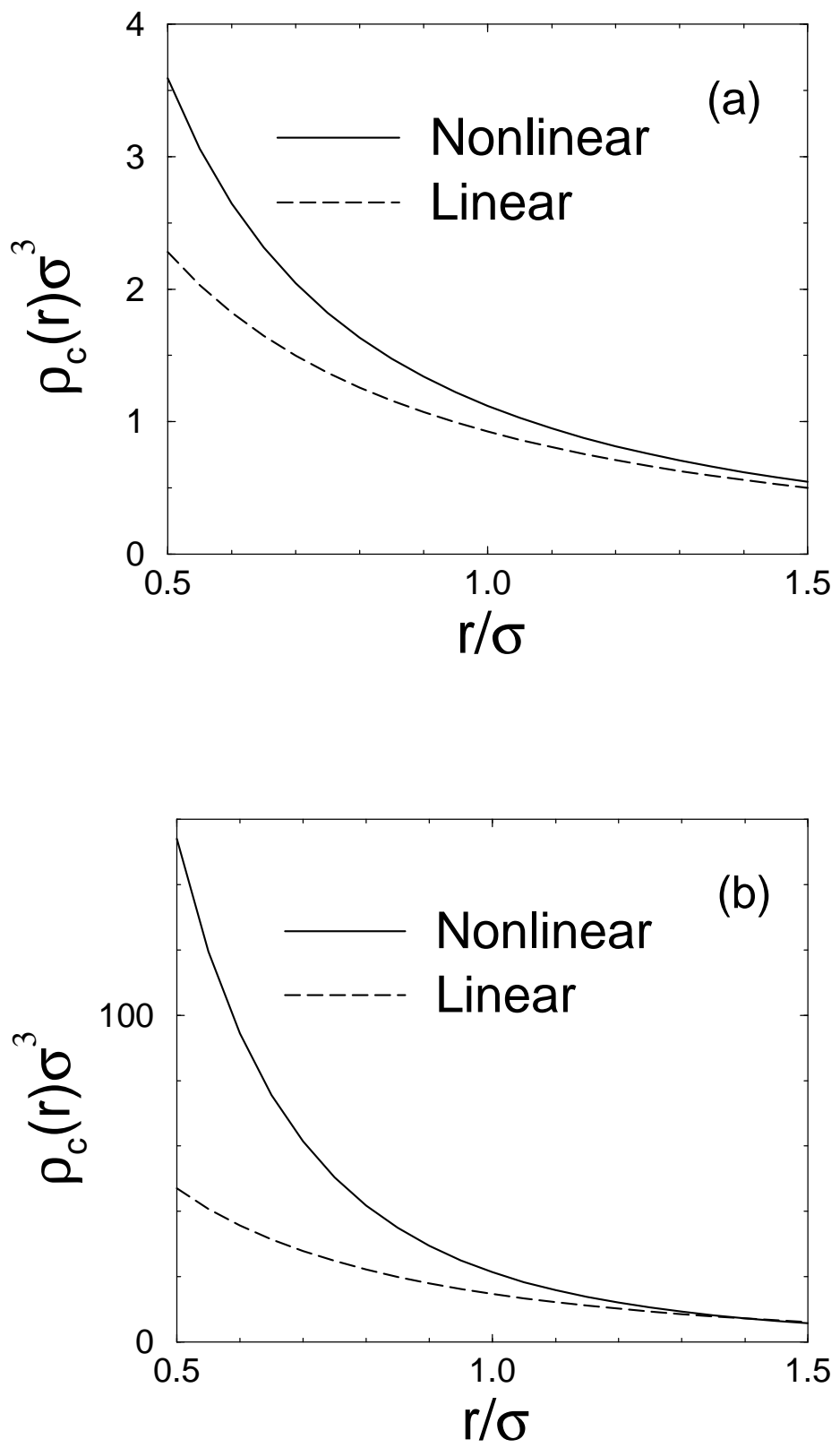

FIG. 3: Ensemble-averaged counterion density around a single macroion for macroion diameter $\sigma=100 \mathrm{~nm}$ and valence (a) $Z=100$, (b) $Z=500$, at volume fraction $\eta=0.01$ and zero salt concentration. Dashed curves: linear response theory. Solid curves: nonlinear response theory (first-order correction). 

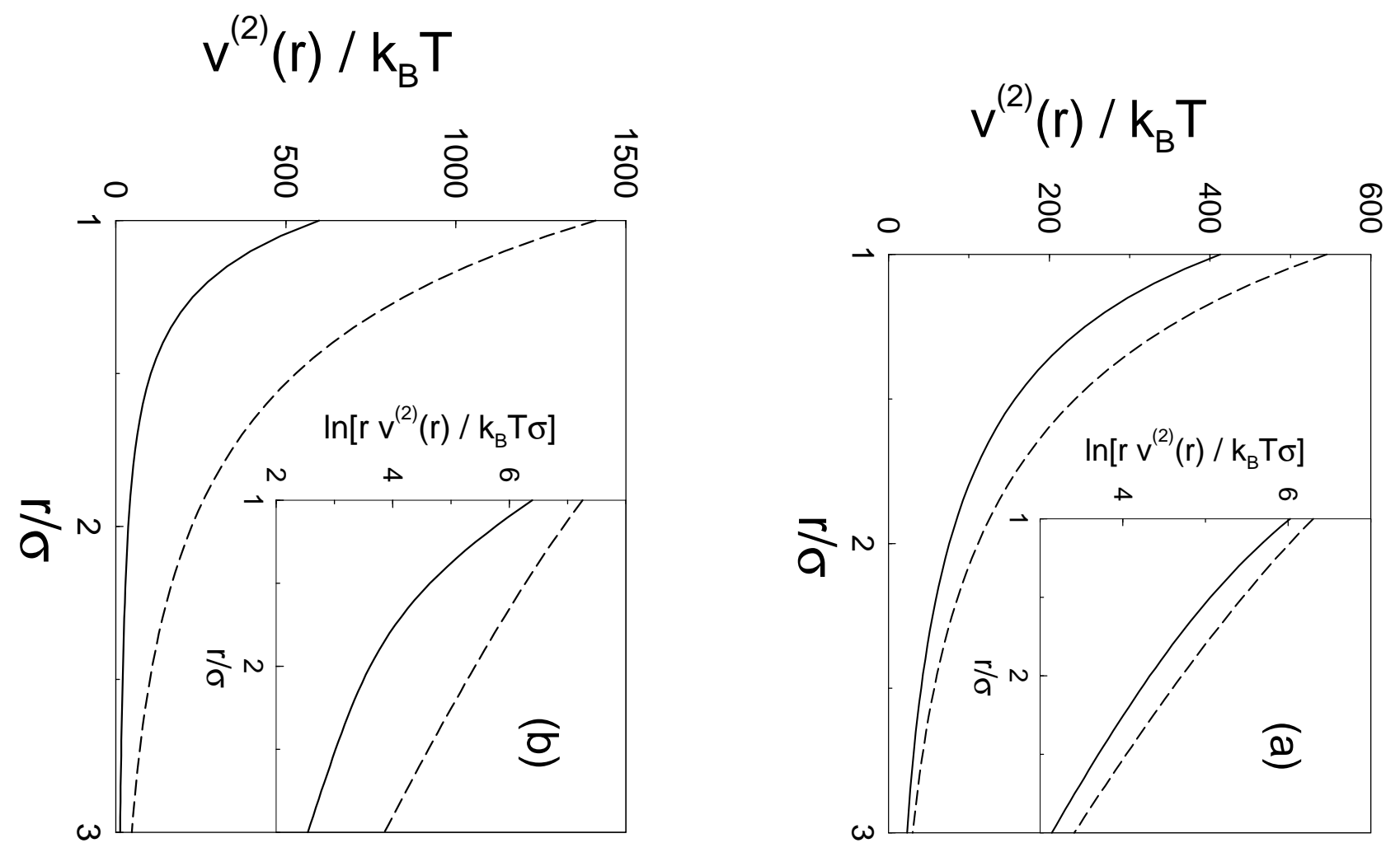


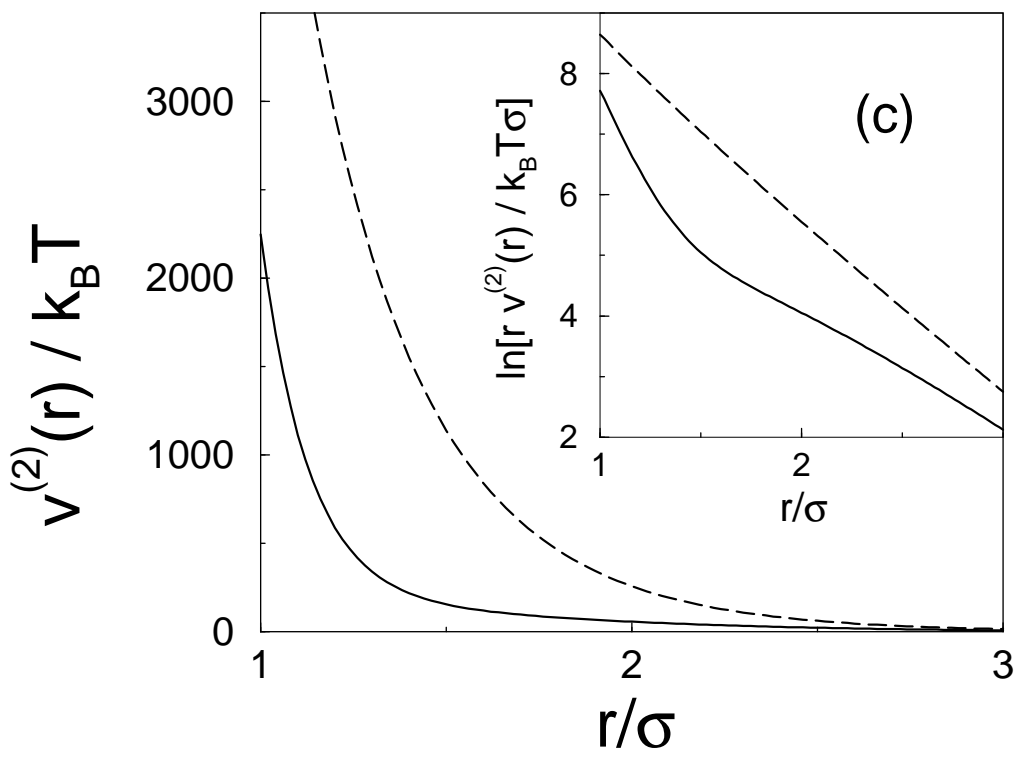

FIG. 4: Effective pair interactions for macroion diameter $\sigma$, valence $Z$, volume fraction $\eta$, and salt concentration $c_{s}$ : (a) $\sigma=100 \mathrm{~nm}, Z=400, \eta=0.01, c_{s}=1 \mu \mathrm{M}$; (b) $\sigma=100 \mathrm{~nm}, Z=700, \eta=0.01, c_{s}=1 \mu \mathrm{M}$; (c) $\sigma=652 \mathrm{~nm}$, $Z=5000, \eta=0.0352, c_{s}=0.2 \mu \mathrm{M}$ (chosen to compare with ref. [10]). Dashed curves: linear response prediction. Solid curves: nonlinear response prediction. The insets show that for sufficiently weakly charged macroions (a) the effective pair interaction may be fit by a Yukawa potential (with a lower effective charge), while for more highly charged macroions (b,c) deviations from linear behavior can be significant. 

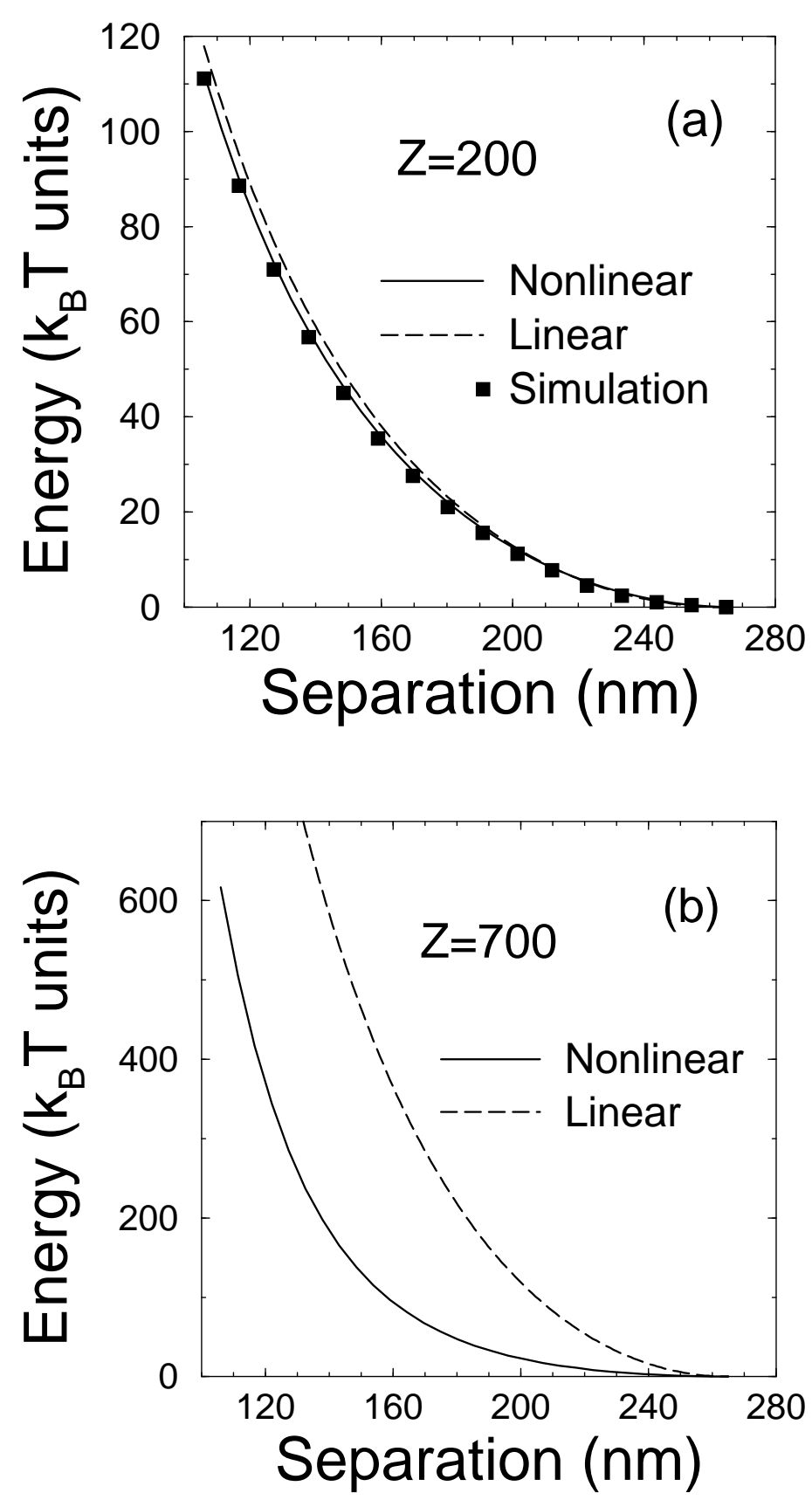

FIG. 5: Total interaction potential energy for two macroions, of diameter $\sigma=106 \mathrm{~nm}$ and valence (a) $Z=200$, (b) $Z=700$, in a cubic box of length $530 \mathrm{~nm}$ with periodic boundary conditions at zero salt concentration. The potentials are shifted to zero at maximum macroion separation. Dashed curves: linear response prediction. Solid curves: nonlinear response prediction. Symbols: ab initio simulation data [21]. 

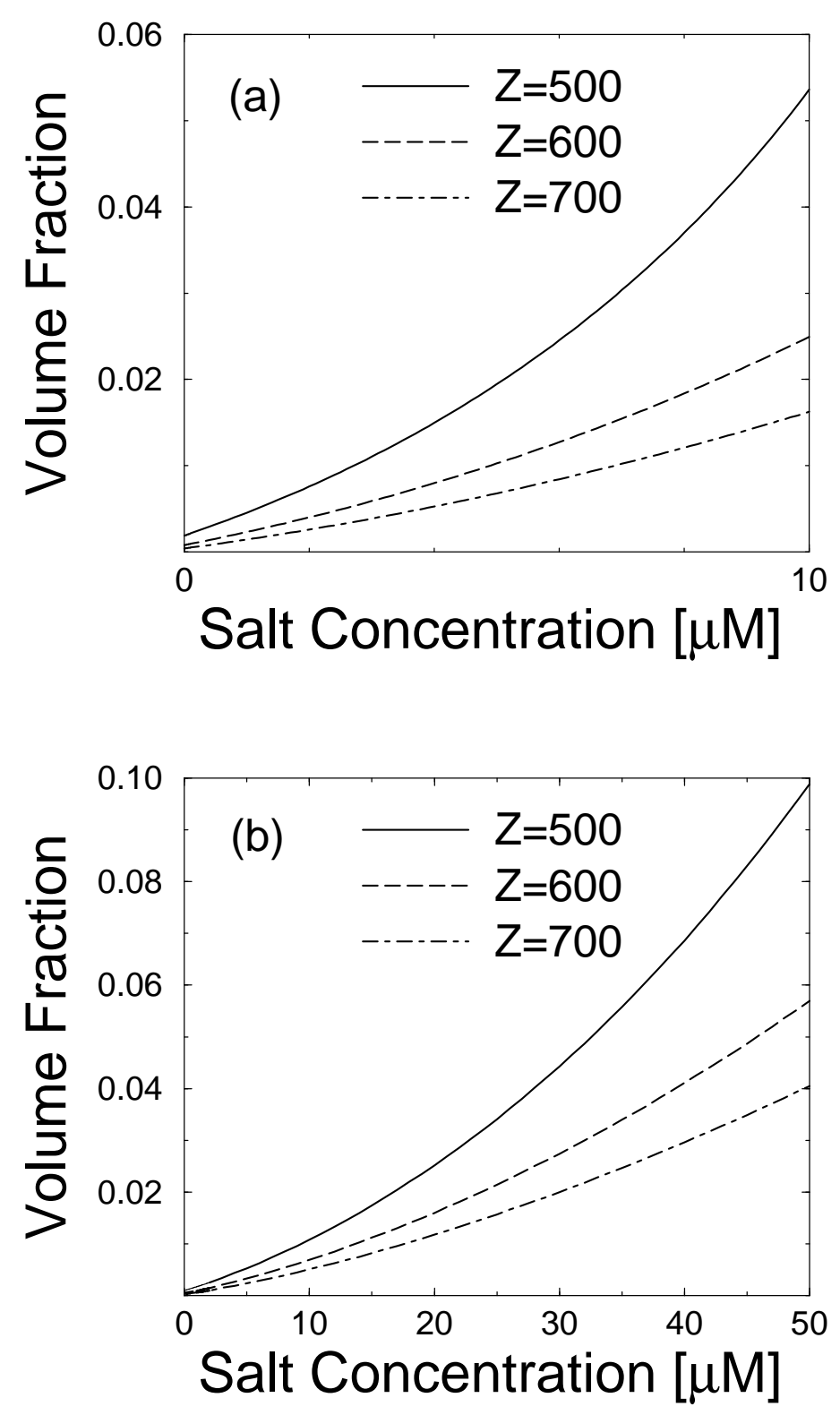

FIG. 6: Map of nonlinear deviations from linear response theory for macroions of diameter $\sigma=100 \mathrm{~nm}$ and valences, from top to bottom, $Z=500,600,700$, for fcc crystal structure. Systems with macroion volume fractions $\eta$ and salt concentrations $c_{s}$ above the respective curves deviate from the linear response pair potential at the fcc nearest-neighbor distance by at least (a) $1 k_{B} T$ or (b) $0.1 k_{B} T$. 


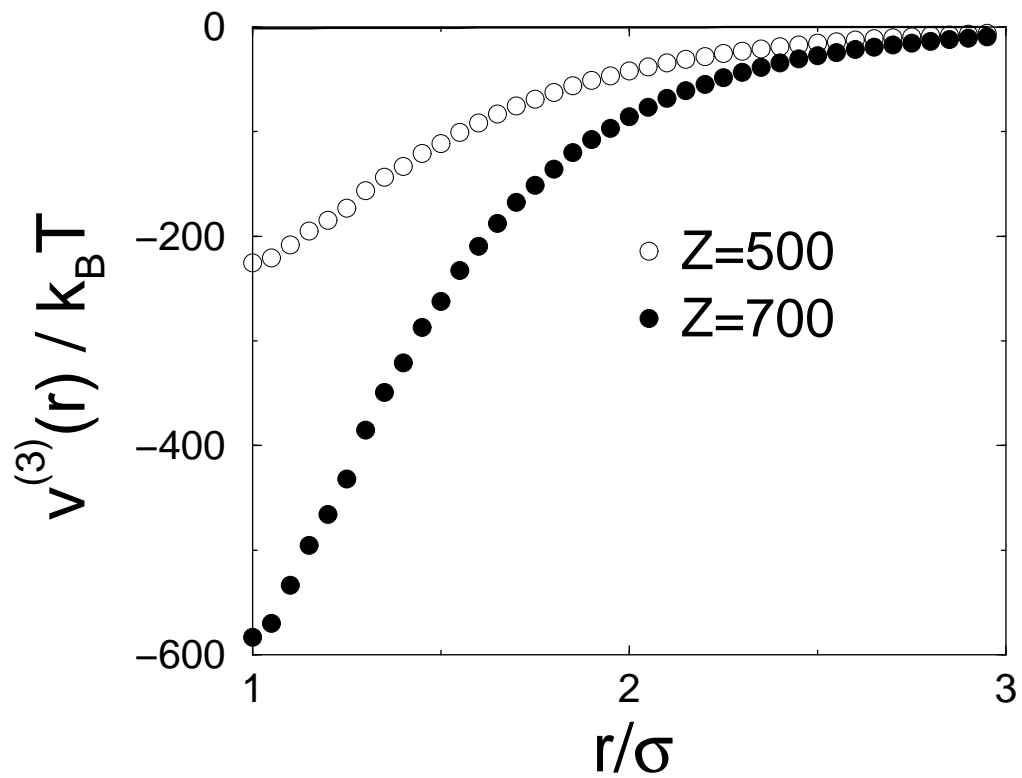

FIG. 7: Effective three-body interaction between three macroions, arranged in an equilateral triangle of side length $r$, with macroion diameter $\sigma=100 \mathrm{~nm}$, valence $Z=500$ (open circles), $Z=700$ (filled circles), volume fraction $\eta=0.01$, and salt concentration $c_{s}=1 \mu \mathrm{M}$. Computed by Monte Carlo integration of Eq. (82), with numerical errors comparable to symbol size. 



FIG. 8: Predicted force on a macroion in an equilateral-triangle arrangement of three macroions, each of diameter $\sigma=106 \mathrm{~nm}$ and valence (a) $Z=700$, (b) $Z=1000$, in a cubic box of length $1000 \mathrm{~nm}$ with periodic boundary conditions at zero salt concentration. Dashed curves: sum of linear response effective pair forces. Solid curves: sum of nonlinear effective pair forces. The symbols are theoretical predictions for the effective triplet force (open circles) and the total (pair plus triplet) effective force (filled circles). 